\title{
Article \\ A Unified Solution for the Vibration Analysis of Lattice Sandwich Beams with General Elastic Supports
}

\author{
Yeqing Jin ${ }^{1} \mathbb{D}$, Ruiping Yang ${ }^{1}$, Hengxu Liu ${ }^{1,2, *}$, Haiwei $\mathrm{Xu}^{2}$ and Hailong Chen ${ }^{1,2}$ \\ 1 Yantai Research Institute and Graduate School, Harbin Engineering University, Yantai 264006, China; \\ jinyeqing@hrbeu.edu.cn (Y.J.); yrp@hrbeu.edu.cn (R.Y.); chenhailong@hrbeu.edu.cn (H.C.) \\ 2 College of Shipbuilding Engineering, Harbin Engineering University, Harbin 150001, China; \\ xhw@hrbeu.edu.cn \\ * Correspondence: 15045630022@163.com
}

Citation: Jin, Y.; Yang, R.; Liu, H.; Xu, H.; Chen, H. A Unified Solution for the Vibration Analysis of Lattice Sandwich Beams with General Elastic Supports. Appl. Sci. 2021, 11, 9141. https://doi.org/10.3390/app11199141

Academic Editor: Jan Awrejcewicz

Received: 17 August 2021

Accepted: 27 September 2021

Published: 1 October 2021

Publisher's Note: MDPI stays neutral with regard to jurisdictional claims in published maps and institutional affiliations.

Copyright: (C) 2021 by the authors Licensee MDPI, Basel, Switzerland. This article is an open access article distributed under the terms and conditions of the Creative Commons Attribution (CC BY) license (https:// creativecommons.org/licenses/by/ $4.0 /)$.

\begin{abstract}
Free vibration analyses of lattice sandwich beams with general elastic supports have rarely been discussed in this field's literature. In this paper, a unified method is proposed to study the free vibration characteristics of lattice sandwich beams under various boundary conditions. The proposed method is to convert the three truss cores of lattice sandwich beams into an equivalent homogeneous layer and introduce two different types of constraint springs to simulate the general elastic support boundary at both ends of lattice sandwich beams. By changing the rigidity of the boundary restraint spring, various boundary conditions can be easily obtained without modifying the solving algorithm and solving process. In order to overcome all the discontinuities or jumps associated with the elastic boundary support conditions, the displacement function of lattice sandwich beams is usually obtained as an improved Fourier cosine series along with four sine terms. On this basis, the unknown series coefficients of the displacement function are treated as the generalized coordinates and solved using the Rayleigh-Ritz method. The correctness of the present method is verified through comparison with existing literature. The calculation results of the present method are highly accurate, indicating that the present method is suitable for analyzing the vibration characteristics of lattice sandwich beams with general elastic supports. In addition, the effects of beam length, panel thickness, core height, radius and truss inclination on the natural frequencies of lattice sandwich beams with arbitrary boundary conditions have been discussed in this paper.
\end{abstract}

Keywords: free vibration; lattice sandwich beams; general elastic supports; improved Fourier series method; Rayleigh-Ritz method

\section{Introduction}

Consisting of two thin panels attached to each side of a thick core, the sandwich structure is a special structure of composite materials. The sandwich structure has been widely used in aviation, aerospace, transportation, ocean engineering and other fields due to its light weight, high rigidity and high strength [1-9]. When the sandwich structure is subjected to a bending load, its panel will bear plane compression and tensile loads, and the core material will bear shear loads. The mechanical properties of the sandwich structure can be obtained by selecting different panel and core material configurations simultaneously. Sandwich structures of foam $[10,11]$ and honeycomb materials $[12,13]$ have been widely used as traditional sandwich structures due to their high rigidity and weight ratio, which can significantly reduce weight while maintaining mechanical properties. However, the conventional sandwich structure cannot be universally used, as it is not compatible with closed-cell foam or a honeycomb core. The space lattice sandwich structure with various types of 3D periodic lattice cores, including pyramidal [14-18], tetrahedral [19,20], Kagome [21,22] and corrugated lattice core [23,24], has been proved to be stronger and more rigid than traditional sandwich structures due to its opening structure with high node connectivity [25]. Based on the developmental trend 
of recent years, the three-dimensional periodic lattice core has been considered the best direction for multifunctional ultra-light structure design.

The space lattice sandwich structure is often dynamically stimulated by the working environment load in practical engineering applications. Therefore, for design purposes, it is of great significance to understand the vibration response mechanism of space lattice sandwich structures. In recent years, a great deal of research has been conducted on the vibration mechanism of space lattice sandwich structures. The most common approach is to transform the sandwich structure as an equivalent homogeneous layer, and then use different panel and shell theories for modeling and calculation analyses. Hwu et al. [26] studied the free vibration and dynamic vibration responses of composite sandwich beams and discussed their influence on transverse shear deformation and rotational inertia. By treating pyramidal truss cores as a continuous homogeneous material, Lou et al. [27] discussed the free vibration characteristic of a sandwich beam under the simply supported boundary condition and verified the calculation results with Abaqus, the finite element analysis software. In order to obtain more a accurate sandwich beam response, Lou et al. [28] introduced a modified piecewise function to establish an analytical model for studying the free vibration response of composite lattice sandwich beams under several typical boundary conditions. Considering the different uncertainties of materials and structures, $\mathrm{Xu}$ and Qiu [29] analyzed the free vibration mechanism and studied the structural optimization of two kinds of space lattice sandwich beams with different core types. Chen et al. [30] selected different panel theories to study the vibration characteristics of composite lattice sandwich panels with three truss cores. By using Hamilton's principle and the assumed mode method and setting up a piezoelectric actuator and sensor on the upper and lower panels of the lattice sandwich beam, $\mathrm{Li}$ and Lyu [31] analyzed the vibration control problem of the beam under a simply supported boundary condition. Li et al.'s work was extended by Zhao et al. [32], who studied the vibration response for a multi-span lattice sandwich beam with a Kagome lattice core and a pyramidal truss core. By applying the Rayleigh-Ritz method, $\mathrm{Xu}$ and his collaborators [33] established an analytical method to study the natural frequency of lattice sandwich beams with a composite gradual corrugated core.

A lot of research on the vibration mechanism of the lattice sandwich beam is basically based on several classical boundary support conditions, including clamped support, free support, simple support and cantilever support. However, there are few studies on general elastic boundary conditions. Li et al. [34-37] first proposed an improved Fourier series method to reveal the vibration mechanisms of beam and plate structures. On this basis, a large number of scholars have put forward many original research results. For a Timoshenko beam with general elastic support conditions, Shi and her team [38] developed a unified method to study their structure response by using virtual springs to simulate arbitrary boundary conditions. By further extending their research, Wang et al. [39] proposed an improved Fourier-Ritz method to analyze the vibration characteristics of composite laminated beams under general boundary conditions. For a Mindlin rectangular plate, Pang et al. [40,41] studied its transverse vibration response by employing the improved Fourier series method with elastic point support boundary conditions. In addition, $\mathrm{Li}$ and Fan [42] studied the bending behavior and local failure of glass fiber-reinforced composite (GFRC) and pultruded sandwich panels (PSPs). Guo et al. [43] were devoted to investigating the sound insulation performance of a simply supported sandwich plate with an hourglass lattice core. Yang et al. [44] experimentally and numerically investigated the free vibration, quasi-static compressive and split Hopkinson pressure bar impact responses of hybrid composite pyramidal truss sandwich panels. Wu et al. [45] revealed elastoplastic deformation and a local indentation failure pattern of the truss-core sandwich panel and material ductility effects to the failure of the truss-core sandwich panel. Sun et al. [46] used the concept of phononic crystals to improve the vibroacoustic properties of truss-core sandwich beams.

However, most of the current research is confined to using classical boundary conditions, and there are few studies on the vibration characteristics of lattice sandwich beams 
under general elastic boundary conditions. In practical engineering, the structure boundary is not an ideal classical boundary condition, but a more general elastic boundary. Therefore, the theoretical analysis method under general elastic boundary support is more conducive to reveal the actual vibration characteristics of the structure. However, the introduction of elastic boundary conditions will lead to a very complicated theoretical modeling process, and the derivative of the vibration differential equation will be discontinuous or jumping. For this reason, this paper presents a unified solution for all boundary conditions, including classical boundary conditions and elastic boundary conditions. In the present method, the truss core of lattice sandwich beams is equivalent to a homogeneous layer, and the lattice sandwich beam is simplified to a laminated beam. By setting tension springs and torsion springs at both ends of the beam, the general elastic support boundary is simulated. Then, the displacement function of lattice sandwich beams is extended to the improved Fourier series expression, and our sine terms are introduced to overcome all the relevant discontinuities or jumps of elastic boundary support conditions. On this basis, the unknown series coefficients of the displacement function are treated as the generalized coordinates and solved using the Rayleigh-Ritz method. The correctness of the present method is verified by comparing with existing literature. The geometric parameters are studied and the vibration characteristics of the structure under elastic boundary conditions are analyzed. This study aims to accumulate data for analyzing the vibration of the lattice sandwich beam.

\section{Theoretical Formulations}

\subsection{Description of the Model}

As shown in Figure 1a,b, a lattice sandwich beam under arbitrary boundary conditions is taken as the research object. In this paper, three different lattice cores are considered, namely, a 3D-Kagome core, a tetrahedral core and a pyramid core, as shown in Figure 2. The coordinate system involved in this paper is shown in Figure $1 \mathrm{a}, \mathrm{b}$, where the $\mathrm{x}-\mathrm{y}$ plane is fixed at the mid-plane of the lattice sandwich beam, the $z$-axis is perpendicular to the $\mathrm{x}-\mathrm{y}$ plane and its direction points downward. The length, width and height of the lattice sandwich beam are defined by $L, B$ and $h$, respectively. The lattice sandwich beam contains a different number of elements in the direction of length and width. Different lengths and widths of sandwich beams can be obtained by selecting different numbers of units.

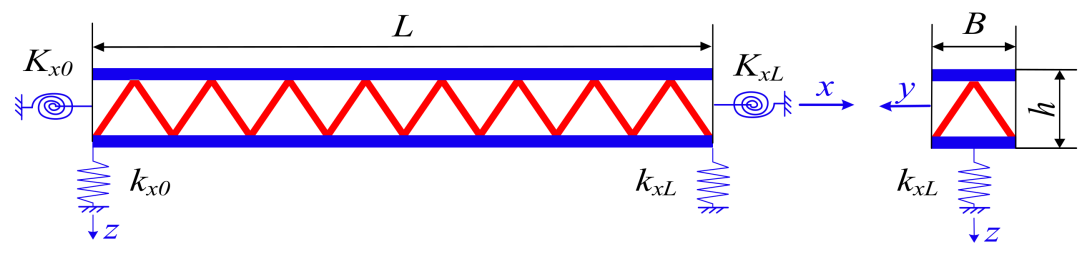

(a)

(b)

Figure 1. Cont. 


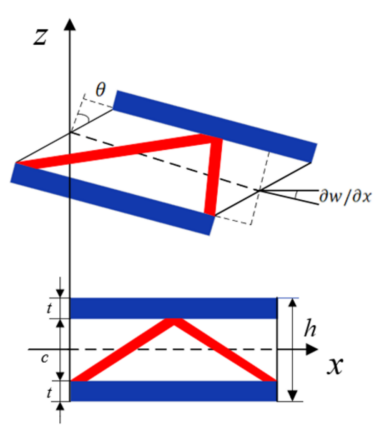

(c)

Figure 1. Schematic diagram of lattice sandwich beams with arbitrary boundary conditions: (a) The structural parameters of the structural system. (b) The cross-sectional view of the structural system. (c) The kinematical parameters of the system.

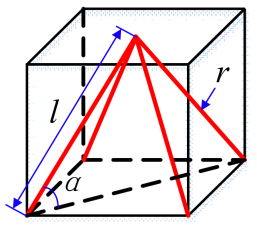

(a)

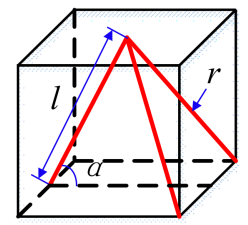

(b)

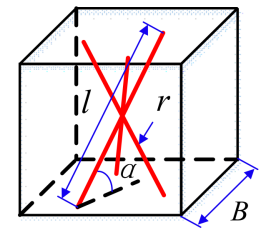

(c)

Figure 2. Schematic diagram of three different lattice core layers. (a) Pyramidal truss core. (b) Tetrahedral truss core. (c) 3D-Kagome truss core.

To explore the vibration mechanism of lattice sandwich beams with arbitrary boundary conditions, the artificial virtual spring technology is first used in this paper. The specific process is to set transverse translational springs and torsion springs at both ends of the beam, based on which the shear force and bending moment at the boundary support can be simulated. Then, by arbitrarily adjusting the rigidity values of the transverse translational springs and torsion springs, arbitrary boundary conditions can be easily simulated. For example, a boundary condition with clamped ends supported can be obtained by setting the rigidity values of the transverse translational springs and the torsion springs at the boundary support to infinity. In practical computations, a maximum number is often used instead of an infinite value. Similarly, setting the rigidity values of these springs at the boundary support to an extremely small value can be considered a free supported boundary.

In theoretical modeling, the upper and lower panels of sandwich beams are thin and hard. Generally, only transverse bending deformation and axial deformation are considered, while shear deformation is ignored. In contrast, the influence of the core layer on the overall flexural rigidity of the lattice sandwich beam is largely ignored because the core layer is generally thicker and lighter. Hence, for a thicker core layer, only the shear deformation is generally taken into account, and the shear stress is considered to be constant in the direction of height. Therefore, based on the above deformation assumptions, the lattice sandwich beam is generally simplified to an equivalent laminated beam composed of the upper and lower panels and an equivalent homogeneous material in the middle layer. Three different lattice cores are involved in this paper, namely, a 3D-Kagome core, a tetrahedral core and a pyramidal core, and are shown in Figure 2. The diameter, length and inclination of the circular truss will be represented by $2 r, l$ and $\alpha$, respectively. According 
to the equivalent principle of mechanics, the equivalent density and equivalent transverse shear modulus of three different core layers are shown as follows [24]:

$$
\begin{gathered}
\text { pyramidal core }: \rho_{c}^{\mathrm{p} y r}=\frac{2 \pi r^{2}}{l^{2} \cos ^{2} \alpha \sin \alpha} \rho, G_{x z c}^{p y r}=\sin \alpha \frac{\pi r^{2}}{l^{2}} E_{s} \\
\text { tetrahedral core }: \rho_{c}^{t e t}=\frac{2 \pi r^{2}}{\sqrt{3} l^{2} \cos ^{2} \alpha \sin \alpha} \rho, G_{x z c}^{t e t}=\sin \alpha \frac{\pi r^{2}}{\sqrt{3} l^{2}} E_{s} \\
\text { 3D-Kagome core }: \rho_{c}^{k a g}=\frac{3 \pi r^{2}}{\sin \alpha B^{2}} \rho, G_{x z c}^{k a g}=\frac{3 \pi \sin \alpha \cos ^{2} \alpha r^{2}}{2 B^{2}} E_{S}
\end{gathered}
$$

where $\rho$ and $E_{\mathrm{s}}$ represent the density and the Young's modulus of the base material, respectively.

\subsection{Energy Functions of Lattice Sandwich Beams}

The analysis in the present paper is limited to linear conditions. Based on the classical sandwich beam theory, the Kirchhoff hypothesis holds, i.e., the straight material line normal to the beam midplane before deformation remains straight and normal to the midplane after deformation. Based on the above deformation hypothesis, the deformation process of the cross-section of the lattice sandwich beam under external force is given Figures $1 \mathrm{c}$ and 3. $H I J K L$ represents a straight line that is normal to the undeformed sandwich beam. If there is no shear strain, $H I J K L$ would rotate by an angle $\partial w / \partial x$ to reach the position $H^{\prime} I^{\prime} J^{\prime} K^{\prime} L^{\prime}$, as shown in Figure 3b. If a shear strain $\theta$ occurs in the core, HIJKL moves to the position $H^{\prime \prime} I^{\prime \prime} J^{\prime \prime} K^{\prime \prime} L^{\prime \prime}$. The lines $H^{\prime \prime} I$ " and $K^{\prime \prime} L^{\prime \prime}$ remain parallel with $H^{\prime} I^{\prime} J^{\prime} K^{\prime} L^{\prime}$, as the shear strains in the face sheets are assumed to be negligible. According to the geometric relationship in the figure, the deformation expression of any point at the cross-section of lattice sandwich beams with different truss cores under arbitrary boundary conditions are given as follows:

$$
\begin{aligned}
& u_{t}=-\frac{c}{2} \theta-z \frac{\partial w}{\partial x}, w_{t}=w,\left(-\frac{h}{2} \leq z \leq-\frac{c}{2}\right) \\
& u_{c}=z\left(\theta-\frac{\partial w}{\partial x}\right), w_{c}=w,\left(-\frac{c}{2} \leq z \leq \frac{c}{2}\right) \\
& u_{b}=\frac{c}{2} \theta-z \frac{\partial w}{\partial x}, w_{b}=w,\left(\frac{c}{2} \leq z \leq \frac{h}{2}\right)
\end{aligned}
$$

where $w$ and $\theta$ represent the lateral displacement of lattice sandwich beams in the $z$-axis direction and the rotation angle in the $y$-axis direction, respectively. The thickness of the upper and lower panels and the height of the core layer are represented by $t$ and $c$, respectively. The horizontal displacements of the upper panel, the core layer and the lower panel in the $x$-axis direction are represented by $u_{\mathrm{t}}, u_{\mathrm{c}}$ and $u_{\mathrm{b}}$, respectively. The lateral displacements of the upper panel, the core layer and the lower panel in the $z$-axis direction are represented by $w_{t}, w_{c}$ and $w_{\mathrm{b}}$, respectively.

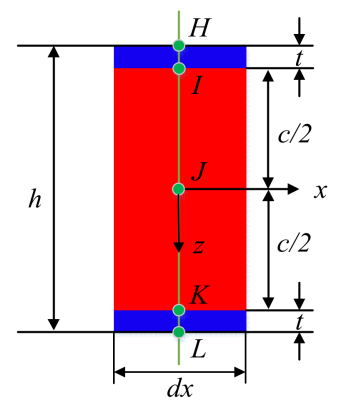

(a)

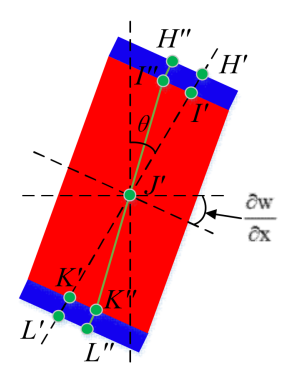

(b)

Figure 3. Deformation of the equivalent lattice sandwich beam. (a) before deformation (b) after deformation. 
According to the above expressions and the definition of the material strain, the strain corresponding to the three layers of the equivalent lattice sandwich beam can be written as the expression of the displacement:

$$
\begin{aligned}
& \varepsilon_{t}=\frac{\partial u_{t}}{\partial x}=-\frac{c}{2} \frac{\partial \theta}{\partial x}-z \frac{\partial^{2} w}{\partial x^{2}},\left(-\frac{h}{2} \leq z \leq-\frac{c}{2}\right) \\
& \gamma_{c}=\frac{\partial u_{c}}{\partial z}+\frac{\partial w}{\partial x}=\theta,\left(-\frac{c}{2} \leq z \leq \frac{c}{2}\right) \\
& \varepsilon_{b}=\frac{\partial u_{b}}{\partial x}=\frac{c}{2} \frac{\partial \theta}{\partial x}-z \frac{\partial^{2} w}{\partial x^{2}},\left(\frac{c}{2} \leq z \leq \frac{h}{2}\right)
\end{aligned}
$$

where $\varepsilon_{t}$ and $\varepsilon_{b}$ represent the normal strain of the upper panel and that of the lower panel, respectively. $\gamma_{c}$ represents the shear strain of the core layer along the length of the beam.

Based on Hooke's Law, the stress corresponding to the three layers of the equivalent lattice sandwich beams can be written as the expression of the displacements:

$$
\begin{aligned}
& \sigma_{t}=E_{x} \varepsilon_{t}=E_{x}\left(-\frac{c}{2} \frac{\partial \theta}{\partial x}-z \frac{\partial^{2} w}{\partial x^{2}}\right),\left(-\frac{h}{2} \leq z \leq-\frac{c}{2}\right) \\
& \tau_{c}=G_{x z c} \theta,\left(-\frac{c}{2} \leq z \leq \frac{c}{2}\right) \\
& \sigma_{b}=E_{x} \varepsilon_{b}=E_{x}\left(\frac{c}{2} \frac{\partial \theta}{\partial x}-z \frac{\partial^{2} w}{\partial x^{2}}\right),\left(\frac{c}{2} \leq z \leq \frac{h}{2}\right)
\end{aligned}
$$

where $E_{\mathrm{x}}$ is the elastic modulus of the upper and lower panels, and $G_{x z c}$ represents the equivalent shear modulus of the core layer. $\sigma_{t}$ and $\sigma_{b}$ represent the normal stress of the upper panel and that of lower panel in the $x$-axis direction, respectively; $\tau_{c}$ represents the shear stress of the core layer.

The next step is to start deriving kinetic and potential energy expressions of the whole structure, obtain the Lagrange energy functional expression and use the Rayleigh-Ritz method to calculate the natural frequency of the structure. The total potential energy $U$ of a lattice sandwich beam under arbitrary boundary conditions consists of two parts: one is the strain energy $U_{B}$ of lattice sandwich beams, and the other is the elastic potential energy $U_{S}$ stored in the restraint springs at both ends of lattice sandwich beams. Their specific expressions can be given as follows:

$$
\begin{gathered}
U=U_{B}+U_{S} \\
\begin{array}{c}
U_{B}=\frac{1}{2} \int_{0}^{L} \int_{A}(\sigma \cdot \varepsilon+\tau \cdot \gamma) d A d x \\
=\frac{B}{2} \int_{0}^{L}\left(\int_{-h / 2}^{-c / 2} \sigma_{t} \varepsilon_{t} d z+\int_{-c / 2}^{c / 2} \tau_{c} \gamma_{c} d z+\int_{c / 2}^{h / 2} \sigma_{b} \varepsilon_{b} d z\right) d x \\
U_{S}=\left.\frac{1}{2} k_{0} w^{2}(x)\right|_{x=0}+\left.\frac{1}{2} K_{0}\left(\frac{\partial w(x)}{\partial x}\right)^{2}\right|_{x=0}+\left.\frac{1}{2} K_{0} \theta^{2}(x)\right|_{x=0} \\
+\left.\frac{1}{2} k_{L} w^{2}(x)\right|_{x=L}+\left.\frac{1}{2} K_{L}\left(\frac{\partial w(x)}{\partial x}\right)^{2}\right|_{x=L}+\left.\frac{1}{2} K_{L} \theta^{2}(x)\right|_{x=L}
\end{array}
\end{gathered}
$$

where $k_{0}$ and $k_{L}$ represent the rigidity constants of the translational springs at the left (where $x=0$ ) and right (where $x=L$ ) ends of lattice sandwich beams, respectively. Similarly, $K_{0}$ and $K_{\mathrm{L}}$ represent the rigidity constants of the torsion springs at the left (where $x=0$ ) and right (where $x=L$ ) ends of the lattice sandwich beams, respectively. For vibration systems, the units of rigidity for the translational and torsional springs are $\mathrm{N} / \mathrm{m}$ and Nm/rad, respectively.

For restraint springs at both ends of the lattice sandwich beam, it is generally assumed that rigidity is provided without considering the effect of their own weight. Additionally, for a lattice sandwich beam under arbitrary boundary conditions, its entire kinetic energy $T$ is determined only by the kinetic energy of the lattice sandwich beam and has nothing to do with the elastic constraints at either end of the beam. At this time, the overall kinetic energy of the system $T$ is expressed as follows: 


$$
\begin{aligned}
& T=\frac{1}{2} \int_{0}^{L} \int_{A} \rho\left[\left(\frac{\partial u}{\partial t}\right)^{2}+\left(\frac{\partial w}{\partial t}\right)^{2}\right] d A d x \\
& =\frac{B}{2} \int_{0}^{L}\left\{\int_{-h / 2}^{-c / 2} \rho_{f}\left[\left(\frac{\partial u_{t}}{\partial t}\right)^{2}+\left(\frac{\partial w_{t}}{\partial t}\right)^{2}\right] d z+\int_{-c / 2}^{c / 2} \rho_{c}\left[\left(\frac{\partial u_{c}}{\partial t}\right)^{2}\right.\right. \\
& \left.\left.+\left(\frac{\partial w_{c}}{\partial t}\right)^{2}\right] d z+\int_{c / 2}^{h / 2} \rho_{f}\left[\left(\frac{\partial u_{b}}{\partial t}\right)^{2}+\left(\frac{\partial w_{b}}{\partial t}\right)^{2}\right] d z\right\} d x
\end{aligned}
$$

where $\rho_{f}$ is the density of the upper panel and lower panel, and $\rho_{c}$ is the equivalent density of the core layer.

\subsection{Displacement Function and Unified Solution}

In order to find the vibration responses of lattice sandwich beams with arbitrary boundary conditions, the improved Fourier series is used to describe the displacement function of the structure in this paper, thereby overcoming the potential discontinuities or jumps of arbitrary boundary conditions during derivation. Hence, the transverse displacement $w$ and the rotation angle $\theta$ of a lattice sandwich beam under arbitrary boundary conditions are expressed as follows [28]:

$$
\begin{gathered}
w(x, t)=\Phi(x) \mathrm{e}^{\mathrm{j} \omega t} \\
\theta(x, t)=\Psi(x) \mathrm{e}^{j \omega t} \\
\Phi(x)=\sum_{m=0}^{\infty} A_{m} \cos \lambda_{m} x+\sum_{n=1}^{4} A_{n}^{a} \sin \lambda_{n} x \\
\Psi(x)=\sum_{m=0}^{\infty} B_{m} \cos \lambda_{m} x+\sum_{n=1}^{4} B_{n}^{a} \sin \lambda_{n} x
\end{gathered}
$$

where $\lambda_{m}=m \pi / L, \lambda_{n}=n \pi / L$ and $\Phi(x)$ and $\Psi(x)$ are the mode shape functions of the lattice sandwich beam under arbitrary boundary conditions, which are described by the improved Fourier series. The improved Fourier series is given as a Fourier cosine series along with four sine terms in this paper. These four sine terms are considered auxiliary functions. $A_{m}$ and $B_{m}$ are the coefficients of the cosine Fourier series, and $A_{n}^{a}$ and $B_{n}^{a}$ are those of the four sine terms, where $n=1,2,3,4$.

As mentioned above, the discontinuity of the original displacement and its derivative can be solved by adding auxiliary functions, and the rapid convergence of the calculation process can also be promoted. Once the structural displacement function is given an explicit expression, the next task is to substitute these displacement function expressions into the kinetic and potential energy expressions. Then, these coefficients in the displacement function are solved by the Rayleigh-Ritz method. The kinetic and potential energy expressions contain the same time term, $\mathrm{e}^{j w t}$, which will also exist after complex derivative and integration operations. For the sake of convenience and simplicity, the time term $\mathrm{e}^{\mathrm{j} w t}$ will be reduced in the following derivation process.

Substituting Equations (2), (3), (8) and (9) into Equation (5) and performing the operation of merging the similar items, the strain energy UB of lattice sandwich beams will be given as:

$$
\begin{gathered}
U_{B}=\frac{B}{2} \int_{0}^{L}\left[\left(A_{\mathrm{t}}+A_{b}\right)\left(\frac{\partial \Psi(x)}{\partial x}\right)^{2}+2\left(B_{t}-B_{b}\right)\left(\frac{\partial \Psi(x)}{\partial x}\right)\right. \\
\left.\cdot\left(\frac{\partial^{2} \Phi(x)}{\partial x^{2}}\right)+\left(D_{\mathrm{t}}+D_{b}\right)\left(\frac{\partial^{2} \Phi(x)}{\partial x^{2}}\right)^{2}+A_{C} \Psi^{2}(x)\right] d x
\end{gathered}
$$

Here

$$
\begin{gathered}
A_{\mathrm{t}}=\left(c^{2} / 4\right) \int_{-h / 2}^{-c / 2} E_{x} d z, B_{\mathrm{t}}=(c / 2) \int_{-h / 2}^{-c / 2} E_{x} z d z, D_{\mathrm{t}}=\int_{-h / 2}^{-c / 2} E_{x} z^{2} d z \\
A_{\mathrm{c}}=\int_{-c / 2}^{c / 2} G_{x z} d z, A_{\mathrm{b}}=\left(c^{2} / 4\right) \int_{c / 2}^{h / 2} E_{x} d z, B_{\mathrm{b}}=(c / 2) \int_{c / 2}^{h / 2} E_{x} z d z, D_{\mathrm{b}}=\int_{c / 2}^{h / 2} E_{x} z^{2} d z
\end{gathered}
$$


Substituting Equations (2), (3), (8) and (9) into Equation (6) and performing the operation of merging the similar items, the potential energy US stored in restraint springs at the two ends of the lattice sandwich beams will be given as:

$$
\begin{aligned}
& U_{S}=\frac{B}{2}\left[\left.k_{0} \Phi^{2}(x)\right|_{x=0}+\left.K_{0}\left(\frac{\partial \Phi(x)}{\partial x}\right)^{2}\right|_{x=0}+\left.K_{0} \Psi^{2}(x)\right|_{x=0}\right. \\
& \left.+\left.k_{L} \Phi^{2}(x)\right|_{x=L}+\left.K_{L}\left(\frac{\partial \Phi(x)}{\partial x}\right)^{2}\right|_{x=L}+\left.K_{L} \Psi^{2}(x)\right|_{x=L}\right]
\end{aligned}
$$

Substituting Equations (2), (3), (8) and (9) into Equation (7) and performing the operation of merging the similar items, the kinetic energy $T$ of the structure will be given as:

$$
\begin{aligned}
& T=\omega^{2} \frac{B}{2} \int_{0}^{L}\left[\left(I_{1}^{\mathrm{t}}+I_{1}^{\mathrm{c}}+I_{1}^{\mathrm{b}}\right) \Psi^{2}(x)+2\left(I_{2}^{\mathrm{t}}-I_{1}^{\mathrm{c}}+I_{2}^{\mathrm{b}}\right)\right. \\
& . \Psi(x)\left(\frac{\partial \Phi(x)}{\partial x}\right)+\left(I_{3}^{\mathrm{t}}+I_{1}^{\mathrm{c}}+I_{3}^{\mathrm{b}}\right)\left(\frac{\partial \Phi(x)}{\partial x}\right)^{2}+ \\
& \left.\left(I_{4}^{\mathrm{t}}+I_{2}^{\mathrm{c}}+I_{4}^{\mathrm{b}}\right) \Phi^{2}(x)\right] d x
\end{aligned}
$$

Here

$$
\begin{gathered}
I_{1}^{\mathrm{t}}=\left(c^{2} / 4\right) \int_{-h / 2}^{-c / 2} \rho_{f} d z, I_{2}^{\mathrm{t}}=(c / 2) \int_{-h / 2}^{-c / 2} \rho_{f} z d z, I_{3}^{\mathrm{t}}=\int_{-h / 2}^{-c / 2} \rho_{f} z^{2} d z, I_{4}^{\mathrm{t}}=\int_{-h / 2}^{-c / 2} \rho_{f} d z, \\
I_{1}^{\mathrm{c}}=\int_{-c / 2}^{c / 2} \rho_{c} z^{2} d z, I_{2}^{\mathrm{c}}=\int_{-c / 2}^{c / 2} \rho_{c} d z, I_{1}^{\mathrm{b}}=\left(c^{2} / 4\right) \int_{c / 2}^{h / 2} \rho_{f} d z, I_{2}^{\mathrm{b}}=(c / 2) \int_{c / 2}^{h / 2} \rho_{f} \mathrm{z} d z, \\
I_{3}^{\mathrm{b}}=\int_{c / 2}^{h / 2} \rho_{f} \mathrm{Z}^{2} d z, I_{4}^{\mathrm{b}}=\int_{c / 2}^{h / 2} \rho_{f} d z
\end{gathered}
$$

and $\omega$ is the angular frequency of the lattice sandwich beam.

Based on the above derivation, the kinetic and potential energy will be shown as the function of unknown coefficients $A_{m}, B_{m}, A_{n}^{a}$ and $B_{n}^{a}$. Thus, the Lagrange energy function $L$ of the structure will be expressed as follows:

$$
L=\left(U_{B}+U_{S}\right)-T
$$

The partial derivatives of the Lagrange energy function for all unknown coefficients $A_{m}, B_{m}, A_{n}^{a}$ and $B_{n}^{a}$ are all calculated by using the standard solution process of the RayleighRitz method. The specific calculation formula is shown as:

$$
\frac{\partial L}{\partial A_{\mathrm{r}}}=0, \frac{\partial L}{\partial A_{s}^{a}}=0, \frac{\partial L}{\partial B_{\mathrm{r}}}=0, \frac{\partial L}{\partial B_{s}^{a}}=0
$$

where $m, r=0 \sim \infty, \mathrm{n}, \mathrm{s}=1,2$.

Substituting Equations (10)-(14) and Equation (13) into Equations (16) and (17), the following can be obtained:

$$
\left(\mathbf{K}-\omega^{2} \mathbf{M}\right) \mathbf{A}=0
$$

where $\mathbf{M}$ and $\mathbf{K}$ are called the mass and rigidity matrices of the structure, respectively; $\mathbf{A}$ denotes the unknown coefficient vector.

According to Equation (17), the eigenvalue equation of lattice sandwich beams can be obtained. Thus, the vibration frequencies and modes of lattice sandwich beams under arbitrary boundary conditions can be calculated by the standard eigenvalue solution method. Equation (17) is described in detail in Appendix A.

\section{Results and Discussions}

\subsection{Validation}

To verify the correctness and accuracy of the present method, this section will first take a pyramidal lattice core sandwich beam as the research object and calculate its natural frequencies under three different typical boundary conditions, including clamped ends, simply supported ends and clamped-free ends. The calculation results in this section will be 
then compared to the data of existing literature, which were calculated by Hwu et al. [19], Lou et al. [21] and $\mathrm{Xu}$ et al. [27] under typical boundary conditions. The material properties and geometric dimensions of the pyramidal lattice sandwich beam involved in the calculation are listed as: $L=0.6364 \mathrm{~m}, B=0.06364 \mathrm{~m}, t=0.0005 \mathrm{~m}, c=0.015 \mathrm{~m}, r=0.001 \mathrm{~m}$, $\alpha=45, E_{s}=E_{x}=210 \mathrm{GPa}, v=0.3$ and $\rho_{f}=\rho=7930 \mathrm{~kg} / \mathrm{m}^{3}$. In order to describe the arbitrary boundary conditions of the lattice sandwich beam, specific abbreviations are used instead of the corresponding boundary conditions. The free, clamped, simply supported and elastic boundary conditions are represented by F, C, SS and E, respectively. By combining them with each other, arbitrary boundary conditions of lattice sandwich beams can be obtained. For example, the symbol C-E can be considered a special case when the lattice sandwich beam is under lamped and elastic boundary conditions at $x=0$ and $x=L$, respectively. For the convenience of calculation, it is necessary to set appropriate rigidity values for these springs and torsional springs to obtain an accurate simulation of the corresponding boundary conditions. Therefore, for clamped ends, the rigidities of these translational and torsion springs at $x=0$ and $x=L$ are all set to a huge value, $10^{14}$. For simply supported ends, the rigidities of the translational springs at $x=0$ and $x=L$ are all set to $10^{14}$, and those of the torsion springs at $x=0$ and $x=L$ are all set to $10^{0}$. For clamped-free ends, the rigidities of the translational and torsion springs at $x=0$ are all set to $10^{14}$, and those of the translational and torsion springs at $x=L$ are all set to $10^{0}$.

According to the above calculation parameters, the first five order natural frequencies of the pyramid lattice sandwich beam under three typical boundary conditions are solved simultaneously. For direct comparison, the calculation results of the present method and the data of existing literature are all listed in Table 1, where the error value is obtained by comparing between Lou's result and the value of the present method. As shown in Table 1, the calculation results in the present method are close to those calculated by Hwu et al. [19], Lou et al. [21] and $\mathrm{Xu}$ et al. [27], indicating that both the theoretical modeling process mentioned in the above section and the programs in the simulation calculations are all correct, and that the present method has sufficient precision. Therefore, this method is very suitable for calculating the free vibration frequency of lattice sandwich beams with arbitrary boundary conditions.

Table 1. Comparison of the natural frequencies for the lattice sandwich beam with a pyramidal lattice core $(\mathrm{Hz})$.

\begin{tabular}{ccccccc}
\hline $\begin{array}{c}\text { Boundary } \\
\text { Condition }\end{array}$ & Mode & $\begin{array}{c}\text { Hwu et al. } \\
{[\text { [19] }}\end{array}$ & $\begin{array}{c}\text { Lou et al. } \\
{[21]}\end{array}$ & $\begin{array}{c}\text { Xu et al. } \\
{[27]}\end{array}$ & $\begin{array}{c}\text { Present Results } \\
\text { (M= 10) }\end{array}$ & Error \\
\hline & 1 & 264.99 & 265.44 & 265.21 & 266.02 & $-0.22 \%$ \\
Clamped & 2 & 693.24 & 695.72 & 694.48 & 699.10 & $-0.49 \%$ \\
ends(CC-CC) & 3 & 1278.8 & 1286.1 & 1282.2 & 1295.25 & $-0.71 \%$ \\
& 4 & 1977.7 & 1993.7 & 1986.9 & 2014.54 & $-1.05 \%$ \\
& 5 & 2756.9 & 2786.3 & 2764.5 & 2817.91 & $-1.13 \%$ \\
\hline Simply & 1 & 121.37 & 121.42 & 121.33 & 121.42 & $0.00 \%$ \\
supported & 2 & 471.66 & 472.30 & 472.1 & 472.31 & $0.00 \%$ \\
ends(SS-SS) & 3 & 1014.9 & 1017.9 & 1014.6 & 1017.95 & $0.00 \%$ \\
& 4 & 1705.6 & 1714.2 & 1702.9 & 1714.18 & $0.00 \%$ \\
\hline & 5 & 2500.0 & 2518.4 & 2506.3 & 2518.40 & $0.00 \%$ \\
\hline Clamped-free & 1 & 43.468 & 43.572 & 43.63 & 43.48 & $0.21 \%$ \\
ends(C-F) & 2 & 265.01 & 266.40 & 265.76 & 265.49 & $0.34 \%$ \\
& 4 & 712.04 & 718.13 & 715.61 & 715.08 & $0.42 \%$ \\
& 5 & 1321.2 & 1337.5 & 1333.7 & 1331.15 & $0.48 \%$ \\
\hline
\end{tabular}

\subsection{Convergence Studies}

According to the above theoretical analysis, the calculation results of this method are mainly related to the rigidity of the constrained spring and the item numbers of the Fourier series. Therefore, this section will focus on the relationship between these factors and the convergence of the present method. It should be noted that the calculation parameters used in the following numerical calculations are the same as those used in the previous 
section. Theoretically, as the number of Fourier series terms increases, the numerical results will become more and more accurate. Therefore, as long as the calculation cost allows it, the present method can be considered to have arbitrary accuracy. However, in the actual calculation process, the Fourier series with infinite terms is often truncated to a finite number of terms $\mathrm{M}$, which ensures the acceptable accuracy of the calculation results.

To investigate the relationship between the item numbers of the Fourier series and the convergence of the present method, the frequency parameter $\Omega=\omega L^{2} \sqrt{\rho A / E I}$ is firstly introduced into this study. Taking the sandwich beam with a pyramidal lattice core under the SS-SS boundary condition as a special case, the first six frequency parameters $\Omega=\omega L^{2} \sqrt{\rho A / E I}$ of the sandwich beam are calculated as the increase of the item numbers (represented by M). The comparison results are shown in Table 2. The results show that the lower the frequency is, the easier the convergence is. When the model sequence (represented by $\mathrm{m}$ ) is less than four, the calculation results can rapidly converge at $\mathrm{M}=6$, and a four-digit calculation precision can be obtained. As the model sequence increases, the number of items that need to be converged also increases. The more items there are, the more accurate the calculation results will be. When the model sequence is greater than 4, the calculation results are plotted in Figure 4a,b, respectively. As shown in Figure 4a,b, when the number of terms is greater than 10 , the results converge stably to all frequencies. Therefore, in order to obtain sufficient accuracy while controlling the calculation cost, all Fourier series will be truncated to $\mathrm{M}=10$ in the following numerical calculations.

Table 2. Convergence analysis of $\Omega=\omega L^{2} \sqrt{\rho A / E I}$ for a SS-SS sandwich beam with a pyramidal lattice core, as $\mathrm{M}$ increases.

\begin{tabular}{ccccccc}
\hline \multirow{2}{*}{$\mathbf{M}$} & \multicolumn{7}{c}{ Model Sequence } \\
\cline { 2 - 7 } & $\mathbf{1}$ & $\mathbf{2}$ & $\mathbf{3}$ & $\mathbf{4}$ & $\mathbf{5}$ & $\mathbf{6}$ \\
\hline 6 & 2.0689 & 8.0478 & 17.3451 & 29.2085 & 42.9238 & 58.0482 \\
8 & 2.0689 & 8.0478 & 17.3451 & 29.2085 & 42.9145 & 57.8734 \\
10 & 2.0689 & 8.0478 & 17.3451 & 29.2085 & 42.9128 & 57.8594 \\
12 & 2.0689 & 8.0478 & 17.3451 & 29.2085 & 42.9118 & 57.8562 \\
14 & 2.0689 & 8.0478 & 17.3451 & 29.2085 & 42.9117 & 57.8560 \\
16 & 2.0689 & 8.0478 & 17.3451 & 29.2085 & 42.9117 & 57.8559 \\
\hline
\end{tabular}

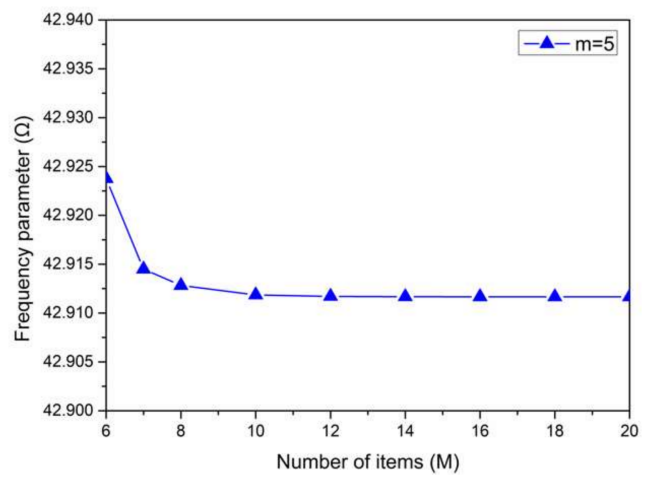

(a) $m=5$

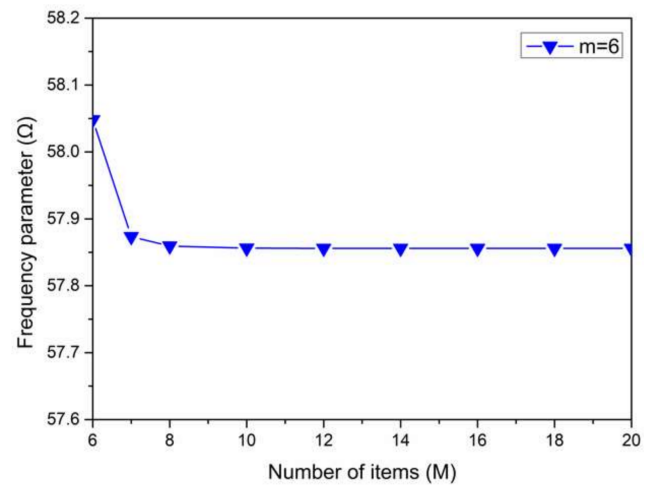

(b) $m=6$

Figure 4. Frequency parameters $\Omega$ for different numbers of items in the lattice sandwich beam with a pyramidal lattice core.

To study the influence of the restraint spring rigidity at both ends of lattice sandwich beams on the convergence of the present method, Figure 5 plots the relationship between the first six order-dimensionless frequency parameters $(\Omega)$ of lattice sandwich beams and the restraint springs' rigidity. During the numerical calculation process, the rigidity of the restraint springs is set in two cases. In one case, based on the simply supported ends (SS-SS), the rigidity of the rotational springs $\left(K_{i}, i=0, L\right)$ gradually increases from $10^{0}$ to 
$10^{14}$. The corresponding results are plotted in Figure $5 \mathrm{a}, \mathrm{b}$. In the other case, the rigidity of the translational springs $\left(k_{i}, i=0, L\right)$ gradually changes from $10^{0}$ to $10^{14}$, when that of the rotational springs is fixed at $10^{14}$. The corresponding results are plotted in Figure $5 c, d$.

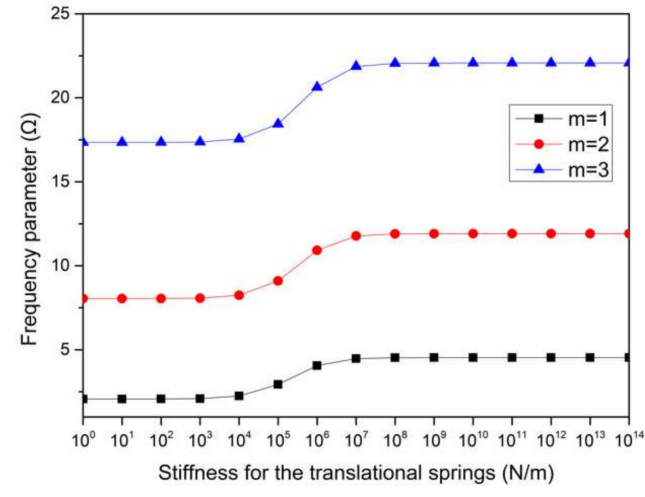

(a) $m=1,2,3$

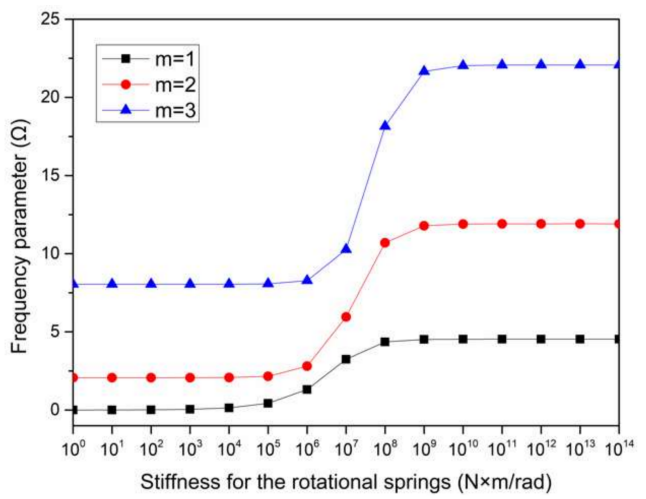

(c) $m=1,2,3$

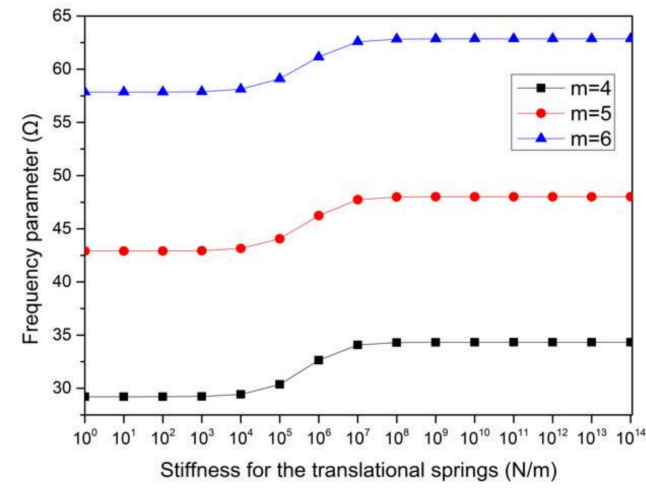

(b) $m=4,5,6$

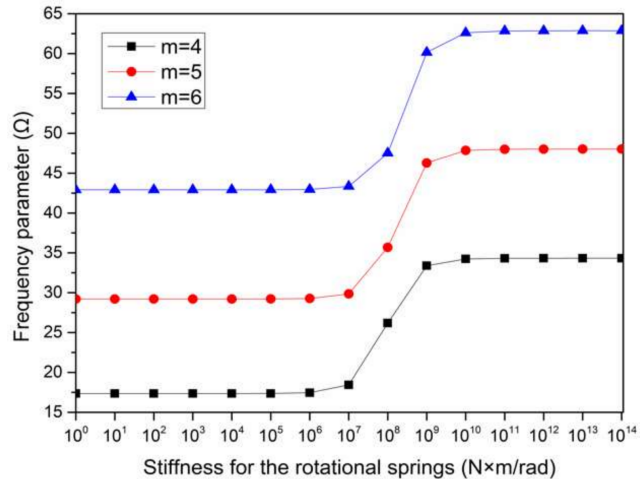

(d) $m=4,5,6$

Figure 5. Relationships of frequency parameters $\Omega$ with restraint spring stiffness of the lattice sandwich beam with a pyramidal lattice core.

As shown in Figure 5, the first six order-dimensionless frequency parameters $(\Omega)$ curve of lattice sandwich beams have the same change rule. As the rigidity for the translational and rotational springs continues to increase, the dimensionless frequency parameters $(\Omega)$ curve of the lattice sandwich beam is a straight line within a certain interval (rigidity changes from $10^{0}$ to $10^{4}$ ), and then it increases sharply within a certain range of rigidity (from $10^{4}$ to $10^{10}$ ) to eventually gradually approach a straight line before remaining unchanged in the range of $10^{10} \sim 10^{14}$. It can be clearly seen that the convergence of the calculation results can be guaranteed when the rigidity value of all types is either more than $10^{10}$ or less than $10^{4}$. The analysis result shows that when the rigidity of the restraint springs is greater than $10^{10}$, it can be regarded as a clamped boundary condition. Afterwards, the increasing rigidity of the restraint springs has little effect on the natural frequency of the lattice sandwich beam. Therefore, the rigidity value equal to $10^{14}$ can be used for simulating infinite rigidity during the numerical calculations. When the rigidity of the restraint springs is less than $10^{4}$, the support effect of the restraint spring is very weak. At that time, it has the same effect as if the rigidity value was equal to zero. It can be regarded as a free boundary condition. Therefore, it is reasonable to use $10^{0}$ to simulate an extremely small rigidity in numerical calculations. These instructions indicate that the constraint spring rigidity set in the previous section is very reasonable for numerical verification. When the rigidity of the restraint springs is between $10^{4}$ and $10^{10}$, it can be regarded as an elastic boundary condition. 
For the convenience of subsequent research, the applicable boundary conditions in the calculation are shown in Table 3, where the symbols F, C and SS, respectively, represent free boundary conditions, clamping boundary conditions and simply supported boundary conditions. The elastic boundary conditions are denoted by E1, E2 and E3. The arbitrary boundary condition can be easily obtained by the different combinations between them.

Table 3. The values of spring rigidity under arbitrary boundary conditions.

\begin{tabular}{ccccccc}
\hline Boundary Conditions & F & C & SS & E1 & E2 & E3 \\
\hline$k i(\mathrm{i}=0, \mathrm{~L})$ & 0 & $10^{14}$ & $10^{14}$ & $10^{5}$ & $10^{6}$ & $10^{7}$ \\
$K i(\mathrm{i}=0, \mathrm{~L})$ & 0 & $10^{14}$ & 0 & $10^{7}$ & $10^{8}$ & $10^{9}$ \\
\hline
\end{tabular}

\subsection{Effects of Boundary Conditions}

To investigate the influence of different boundary conditions on the vibration characteristics of lattice sandwich beams, twenty-one different boundary conditions are first obtained from Table 3 by free combination. They are divided into three different groups. The first group includes F-F, SS-SS, C-C, SS-F, C-F and SS-C, which represent classical boundary conditions. The second one includes E1-E1, E-E2, E3-E3, E1-E2, E1-E3 and E2-E3, which represent elastic boundary conditions. The third one includes E1-F, E2-F, E3-F, E1-C, E2-C, E3-C, E1-SS, E2-SS and E3-SS, which represent arbitrary boundary conditions. To clarify the meaning of the above symbols, the symbol E-SS will be described in detail as an example; that is, at $x=0$ and $x=L$, the boundary supports of the sandwich beam are elastic supports and simple supports, respectively. In this case, $k_{0}=10^{6}, K_{0}=10^{8}$ at $x=0$ and $k_{L}=10^{14}, K_{L}=0$ at $x=L$. Based on these three different groups of boundary conditions, the first six order dimensionless frequency parameters $(\Omega)$ of lattice sandwich beams under the corresponding boundary conditions are shown in Tables 4-6, respectively.

Table 4. Frequency parameters $\Omega$ for lattice sandwich beams with several classical boundary conditions.

\begin{tabular}{ccccccc}
\hline \multirow{2}{*}{ Boundary Conditions } & \multicolumn{7}{c}{ Mode Sequence } \\
\cline { 2 - 7 } & $\mathbf{1}$ & $\mathbf{2}$ & $\mathbf{3}$ & $\mathbf{4}$ & $\mathbf{5}$ & $\mathbf{6}$ \\
\hline F-F & 0.0000 & 0.0019 & 4.6697 & 12.4591 & 23.3257 & 36.4479 \\
SS-SS & 2.0689 & 8.0478 & 17.3451 & 29.2085 & 42.9118 & 57.8562 \\
C-C & 4.5327 & 11.9122 & 22.0701 & 34.3264 & 48.0153 & 62.8635 \\
SS-F & 0.0009 & 3.2241 & 10.1398 & 20.2331 & 32.7395 & 46.9466 \\
C-F & 0.7408 & 4.5238 & 12.1844 & 22.6818 & 35.3457 & 49.5428 \\
SS-C & 3.1828 & 9.9286 & 19.7000 & 31.7739 & 45.5013 & 60.3664 \\
\hline
\end{tabular}

Table 5. Frequency parameters $\Omega$ for lattice sandwich beams with several elastic boundary conditions.

\begin{tabular}{ccccccc}
\hline \multirow{2}{*}{ Boundary Conditions } & \multicolumn{7}{c}{ Mode Sequence } \\
\cline { 2 - 7 } & $\mathbf{1}$ & $\mathbf{2}$ & $\mathbf{3}$ & $\mathbf{4}$ & $\mathbf{5}$ & $\mathbf{6}$ \\
\hline E1-E1 & 0.4264 & 2.1411 & 8.0147 & 17.2445 & 29.0461 & 42.6961 \\
E2-E2 & 1.3112 & 2.8063 & 8.2706 & 17.4394 & 29.2536 & 42.9313 \\
E3-E3 & 3.2448 & 5.9553 & 10.2655 & 18.4350 & 29.8442 & 43.3382 \\
E1-E2 & 0.8600 & 2.5327 & 8.1444 & 17.3421 & 29.1498 & 42.8136 \\
E1-E3 & 1.2028 & 4.5804 & 9.3204 & 17.8623 & 29.4499 & 43.0185 \\
E2-E3 & 1.7983 & 4.7050 & 9.4148 & 17.9553 & 29.5525 & 43.1358 \\
\hline
\end{tabular}


Table 6. Frequency parameters $\Omega$ for lattice sandwich beams with arbitrary boundary conditions.

\begin{tabular}{ccccccc}
\hline \multirow{2}{*}{ Boundary Conditions } & \multicolumn{7}{c}{ Mode Sequence } \\
\cline { 2 - 7 } & $\mathbf{1}$ & $\mathbf{2}$ & $\mathbf{3}$ & $\mathbf{4}$ & $\mathbf{5}$ & $\mathbf{6}$ \\
\hline E1-F & 0.2869 & 1.2339 & 6.2327 & 14.7937 & 26.1570 & 39.5668 \\
E2-F & 0.6171 & 1.7714 & 6.3907 & 14.8940 & 26.2590 & 39.6822 \\
E-3F & 0.7272 & 3.6098 & 7.8701 & 15.5133 & 26.5960 & 39.9047 \\
E1-C & 1.2617 & 6.1255 & 14.4416 & 25.4206 & 38.3692 & 52.6972 \\
E2-C & 1.8772 & 6.2805 & 14.5400 & 25.5202 & 38.4800 & 52.8244 \\
E3-C & 3.6773 & 7.7494 & 15.1622 & 25.8595 & 38.7019 & 52.9903 \\
E1-SS & 0.6716 & 4.6038 & 12.2935 & 22.9374 & 35.7759 & 50.1410 \\
E2-SS & 1.3834 & 4.8051 & 12.3983 & 23.0363 & 35.8864 & 50.2658 \\
E3-SS & 2.7371 & 6.5917 & 13.1531 & 23.4202 & 36.1301 & 50.4421 \\
\hline
\end{tabular}

Comparing the data in Tables 4-6, it can be clearly concluded that the influence of boundary conditions on structural vibration is very important. When the boundary conditions are different, there will be a large difference in the frequency parameters $(\Omega)$ of the lattice sandwich. As the rigidity of the restraint spring at both ends of the lattice sandwich beam continues to increase, its frequency parameters $(\Omega)$ also increase. Among them, the frequency parameters $(\Omega)$ are the largest under the lamped ends. These results have accumulated data for discussing the vibration characteristics of lattice sandwich beams under arbitrary boundary conditions.

In order to further study the influence of boundary conditions on the vibration characteristics of lattice sandwich beams, the mode shapes of lattice sandwich beams under different boundary conditions are also plotted. To facilitate this study, one of the three different boundary conditions mentioned above is selected as the representative, namely, SS-SS, E3-E3 and E3-SS, and its mode shape is drawn in Figures 6-8, respectively. Comparing Figures $6-8$, it can be seen that the mode shapes of the structure under the three boundary conditions are different. The boundary conditions have a significant influence on the mode shape of the structure. Under the classical boundary conditions (e.g., SS-SS), the number of peaks and troughs in the mode shapes of lattice sandwich beams has a one-to-one relationship with the modal orders of the structure. That is, one mode shape corresponds to a peak or trough. However, under elastic boundary support conditions, this correspondence is not obvious. The elastic boundary support reduces the node effect of the boundary support.
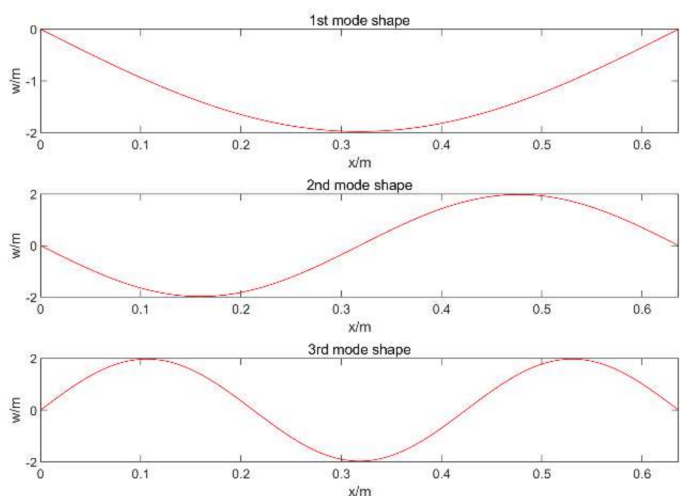

(a)

Figure 6. Three initial mode shapes of the SS-SS lattice sandwich beam. (a) First mode shape, (b) Second mode shape and (c) Third mode shape. 


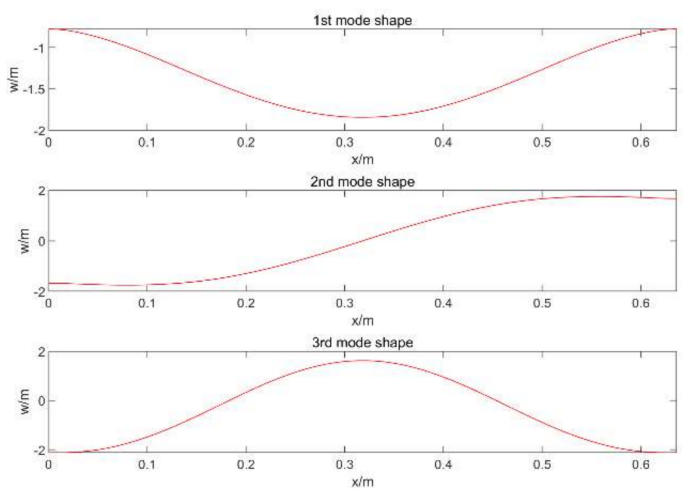

(a)

(b)

(c)

Figure 7. Three initial mode shapes of the E3-E3 lattice sandwich beam. (a) First mode shape, (b) Second mode shape and (c) Third mode shape.
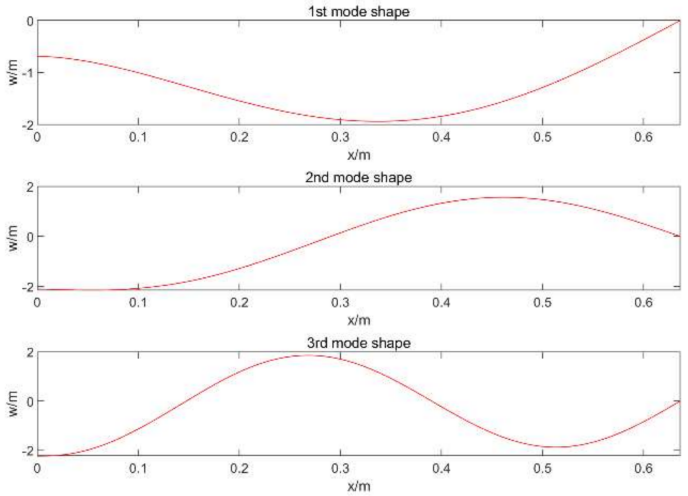

(a)

(b)

(c)

Figure 8. Three initial mode shapes of the E3-SS lattice sandwich beam. (a) First mode shape, (b) Second mode shape and (c) Third mode shape.

\subsection{Effect of Geometric Parameters}

For pyramidal truss-core sandwich beams, the first natural frequencies of structures under different boundary conditions, including SS-SS, C-C, E3-E3, E3-SS and E3-C, are calculated by changing the geometric parameters such as beam length, plate thickness and core height. The calculation results are listed in Tables 7-9, respectively. For comparison, the data shown in Tables 7-9 are also plotted in Figures 9-11, respectively.

Table 7. Comparison of the natural frequencies of pyramidal truss-core sandwich beams of different beam lengths under arbitrary boundary conditions $(\mathrm{Hz})$.

\begin{tabular}{cccccc}
\hline \multirow{2}{*}{ Cell Numbers } & \multicolumn{5}{c}{ Arbitrary Boundary Conditions } \\
\cline { 2 - 6 } & SS-SS & C-C & E3-E3 & E3-SS & E3-C \\
\hline 15 & 472.3090 & 951.5135 & 337.8441 & 349.2931 & 459.7782 \\
20 & 269.9761 & 569.5114 & 278.0257 & 267.9476 & 343.5740 \\
25 & 174.1084 & 376.1836 & 230.4454 & 207.4917 & 270.8658 \\
30 & 121.4175 & 266.0154 & 190.4328 & 160.6320 & 215.8123 \\
35 & 89.4326 & 197.6274 & 156.9436 & 125.4781 & 172.5897 \\
40 & 68.5858 & 152.4518 & 129.5811 & 99.5839 & 139.1626 \\
45 & 54.2533 & 121.0526 & 107.6502 & 80.4497 & 113.5873 \\
\hline
\end{tabular}


Table 8. Comparison of the natural frequencies of pyramidal truss-core sandwich beams of different panel thicknesses under arbitrary boundary conditions $(\mathrm{Hz})$.

\begin{tabular}{cccccc}
\hline \multirow{2}{*}{$\begin{array}{c}\text { Panel Thicknesses } \\
(\mathbf{m m})\end{array}$} & \multicolumn{5}{c}{ Arbitrary Boundary Conditions } \\
\cline { 2 - 6 } & SS-SS & C-C & E3-E3 & E3-SS & E3-C \\
\hline 0.5 & 121.4175 & 266.0154 & 190.4328 & 160.6320 & 215.8123 \\
1 & 137.7297 & 292.6634 & 168.9821 & 155.7359 & 201.3014 \\
1.5 & 146.5784 & 302.9722 & 150.4007 & 145.0440 & 185.5933 \\
2 & 153.0482 & 308.5381 & 136.3312 & 135.3830 & 173.7080 \\
2.5 & 158.4541 & 312.3427 & 125.4501 & 127.4007 & 164.9065 \\
3 & 163.2901 & 315.3960 & 116.7719 & 120.8833 & 158.3260 \\
3.5 & 167.7836 & 318.1906 & 109.6617 & 115.5383 & 153.3543 \\
\hline
\end{tabular}

Table 9. Comparison of the natural frequencies of pyramidal truss-core sandwich beams of different core heights under arbitrary boundary conditions $(\mathrm{Hz})$.

\begin{tabular}{cccccc}
\hline \multirow{2}{*}{$\begin{array}{c}\text { Core Height } \\
(\mathbf{m m})\end{array}$} & SS-SS & C-C & E3-E3 & E3-SS & E3-C \\
\cline { 2 - 6 } & 32.9684 & 74.7245 & 70.7994 & 50.4886 & 72.6211 \\
5 & 76.0519 & 170.5798 & 142.6226 & 110.1543 & 154.1021 \\
10 & 121.4175 & 266.0154 & 190.4328 & 160.6320 & 215.8123 \\
15 & 166.4761 & 349.9606 & 218.4888 & 197.0480 & 256.0741 \\
20 & 209.5006 & 415.6572 & 234.9471 & 221.5729 & 282.5575 \\
25 & 249.0683 & 461.2186 & 244.8919 & 238.1765 & 300.9681 \\
30 & 284.0671 & 488.7007 & 251.0252 & 249.8877 & 314.1467 \\
35 & \multicolumn{5}{c}{} \\
\hline
\end{tabular}

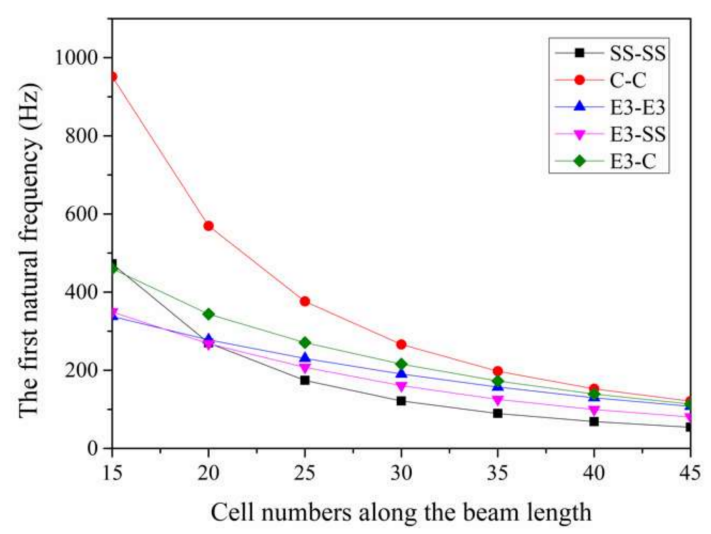

Figure 9. Effect of the beam length on the first natural frequency of the pyramidal truss-core sandwich beam under arbitrary boundary conditions.

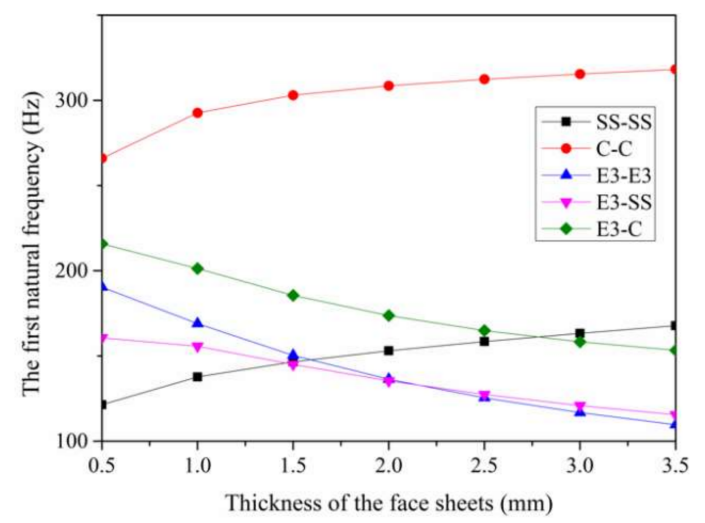

Figure 10. Effect of the panel thickness on the first natural frequency of the pyramidal truss-core sandwich beam under arbitrary boundary conditions. 


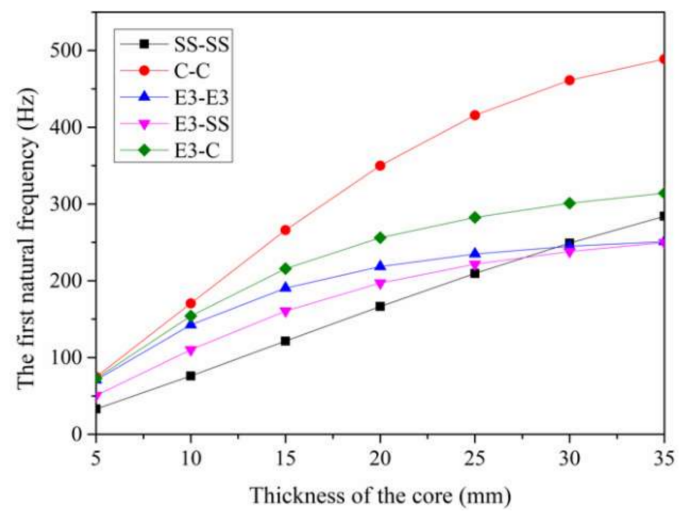

Figure 11. Effect of the core height on the first natural frequency of the pyramidal truss-core sandwich beam under arbitrary boundary conditions.

It can be seen from Table 7 and Figure 9 that, for different boundary conditions, the free vibration frequency of the structure decreases with the increase of the cell number. The main reason is that as the beam length continues to increase, the rigidity of the structure decreases due to the increase in its weight. Combining Table 8 and Figure 10, it can be known that the free vibration frequencies of the structure will become larger due to the increase of the panel thickness under C-C and SS-SS boundary conditions, but it will become smaller as the panel thickness increases under E3-E3, E3-SS and E3-C boundary conditions. The main reason is that when the boundary conditions are C-C and SS-SS, the rigidity of the restraint springs at both ends of the lattice sandwich beam is sufficiently large. In this case, the increase in the bending rigidity of the structure is greater than that in the structural weight. As a result, the free vibration frequencies of the structure will increase as the panel thickness increases. However, when the boundary conditions are E3-E3, E3-SS and E3-C, the overall rigidity of the restraint springs at boundary supports is relatively small. In this case, the increase in structural weight is dominant, but the increase in structural bending rigidity is relatively small. Hence, the free vibration frequencies of the structure will decrease as the panel thickness increases. These statements indicate that the boundary support conditions have an important effect on the free vibration frequency of lattice sandwich beams, especially under the elastic boundary conditions.

It can be seen from Table 9 and Figure 11 that, under different boundary conditions, with the increase of core height, the free vibration frequency of lattice sandwich beams will increase. The main reason is that as the core height continues to increase, the equivalent density of the truss core will decrease, resulting in a decline in the weight of the structure and an increase in its natural frequency. In addition, comparing Figures 10 and 11, under the classical boundary conditions such as C-C and SS-SS, it is more effective to change the free vibration frequencies of lattice sandwich beams by changing the core height than by changing the panel thickness because, when the height of the core layer increases, not only is the weight of the structure reduced, but also the moment of inertia of the structure increases.

\subsection{Effects of Different Truss Cores}

In this section, the free vibration characteristics of the lattice sandwich beam with three different truss cores, including 3D-Kagome truss core, tetrahedral truss core and pyramidal truss core, whose will be discussed. The free vibration frequencies of three kinds of truss core structures under arbitrary boundary conditions (SS-SS, C-C, E3-E3, E3-SS and E3-C) are calculated by changing the geometrical parameters such as radius and inclination of the truss Tables 10-12 give the curve of the free vibration frequencies of the structure with different truss radius. For comparison, the results listed in Tables 10-12 are also displayed in Figures 12-14, respectively. Tables 13-15 gives the curve of the free vibration frequencies of the structure with different inclination angles. For comparison, the results listed in Tables 13-15 are also displayed in Figures 17-19, respectively. 
Table 10. Comparisons of the natural frequencies of pyramidal truss-core sandwich beams with different truss radius under arbitrary boundary conditions $(\mathrm{Hz})$.

\begin{tabular}{cccccc}
\hline \multirow{2}{*}{$\begin{array}{c}\text { Truss Radius } \\
(\mathbf{m m})\end{array}$} & SS-SS & C-C & E3-E3 & E3-SS & E3-C \\
\cline { 2 - 6 } & 139.9091 & 267.8756 & 208.1397 & 177.9489 & 230.7810 \\
0.4 & 136.8992 & 284.5443 & 210.5608 & 178.4970 & 236.6766 \\
0.6 & 129.8016 & 279.3969 & 202.2866 & 170.9090 & 228.6271 \\
0.8 & 121.4175 & 266.0154 & 190.4328 & 160.6320 & 215.8123 \\
1 & 112.8727 & 249.7781 & 177.6603 & 149.7228 & 201.6419 \\
1.2 & 104.6991 & 233.1444 & 165.1523 & 139.1051 & 187.6189 \\
1.4 & 97.1334 & 217.1685 & 153.4348 & 129.1895 & 174.4136 \\
1.6 & 90.2537 & 202.3523 & 142.7060 & 120.1265 & 162.2863 \\
1.8 & 84.0553 & 188.8378 & 132.9978 & 111.9348 & 151.2920 \\
2 & & & & & \\
\hline
\end{tabular}

Table 11. Comparisons of the natural frequencies of tetrahedral truss-core sandwich beams with different truss radii under arbitrary boundary conditions $(\mathrm{Hz})$.

\begin{tabular}{cccccc}
\hline \multirow{2}{*}{$\begin{array}{c}\text { Radius of } \\
\text { the Truss }(\mathbf{m m})\end{array}$} & SS-SS & C-C & E3-E3 & E3-SS & E3-C \\
\cline { 2 - 6 } & 137.4313 & 242.6776 & 197.1684 & 170.1449 & 215.5373 \\
0.4 & 139.8083 & 276.1559 & 210.7334 & 179.5651 & 234.8861 \\
0.6 & 136.6687 & 284.5942 & 210.3639 & 178.2960 & 236.5277 \\
0.8 & 131.3832 & 281.3776 & 204.3654 & 172.7485 & 230.7951 \\
1 & 125.1822 & 272.4854 & 195.8814 & 165.3260 & 221.7693 \\
1.2 & 118.6770 & 261.0067 & 186.3849 & 157.1637 & 211.3455 \\
1.4 & 112.2116 & 248.4577 & 176.6567 & 148.8691 & 200.5202 \\
1.6 & 105.9816 & 235.7920 & 167.1272 & 140.7789 & 189.8390 \\
1.8 & 100.0916 & 223.4619 & 158.0284 & 133.0740 & 179.5965 \\
2 & & & & & \\
\hline
\end{tabular}

Table 12. Comparisons of the natural frequencies of 3D-Kagome truss-core sandwich beams with different truss radii under arbitrary boundary conditions $(\mathrm{Hz})$.

\begin{tabular}{cccccc}
\hline \multirow{2}{*}{$\begin{array}{c}\text { Radius of } \\
\text { the Truss }(\mathbf{m m})\end{array}$} & SS-SS & C-C & E3-E3 & E3-SS & E3-C \\
\cline { 2 - 6 } & 139.0700 & 255.8957 & 203.3113 & 174.5988 & 223.8548 \\
0.4 & 138.8832 & 281.7456 & 211.6004 & 179.8111 & 236.9097 \\
0.6 & 133.8783 & 283.8063 & 207.4455 & 175.5245 & 233.8970 \\
0.8 & 127.1032 & 275.5349 & 198.5927 & 167.6778 & 224.6977 \\
1 & 119.7316 & 262.9310 & 187.9493 & 158.5026 & 213.0750 \\
1.2 & 112.3501 & 248.7202 & 176.8671 & 149.0480 & 200.7555 \\
1.4 & 105.2674 & 234.2966 & 166.0278 & 139.8470 & 188.6031 \\
1.6 & 98.6351 & 220.3493 & 155.7685 & 131.1625 & 177.0474 \\
1.8 & 92.5132 & 207.2480 & 146.2359 & 123.1070 & 166.2793 \\
\hline
\end{tabular}




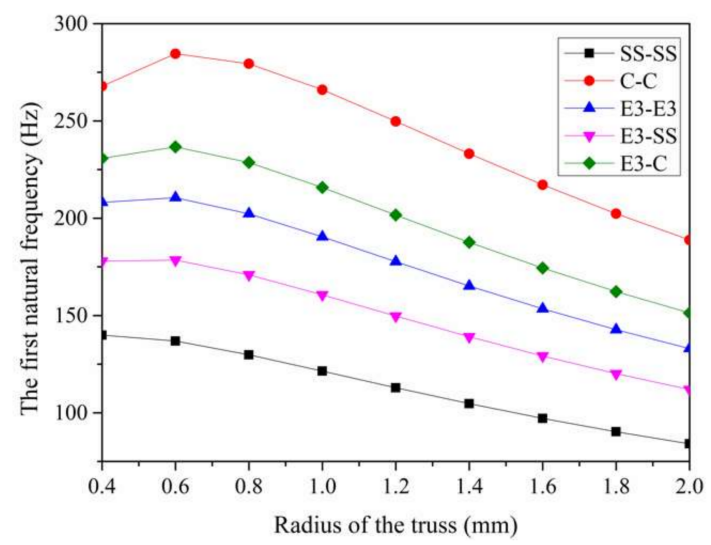

Figure 12. Effect of the truss radius on the first natural frequency of the pyramidal truss-core sandwich beam under arbitrary boundary conditions.

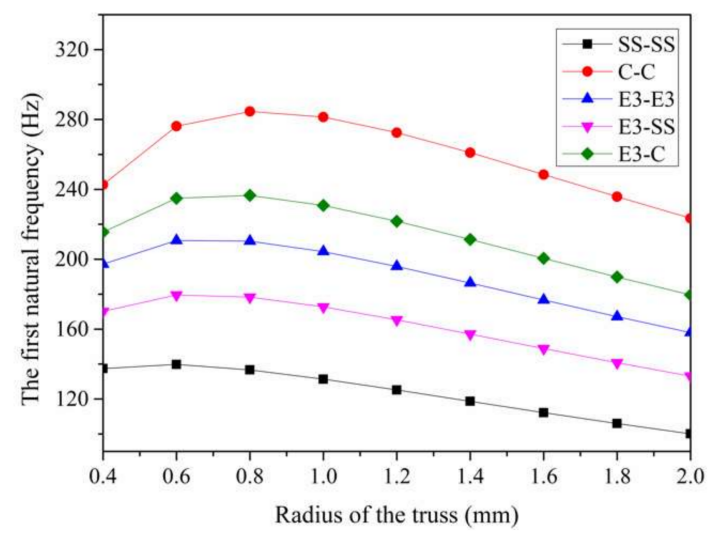

Figure 13. Effect of the truss radius on the first natural frequency of the tetrahedral truss-core sandwich beam under arbitrary boundary conditions.

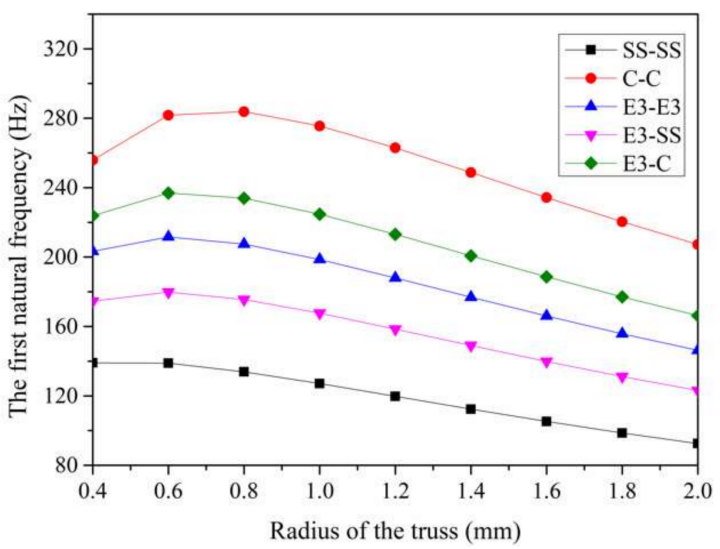

Figure 14. Effect of the truss radius on the first natural frequency of the 3D-Kagome truss-core sandwich beam under arbitrary boundary conditions. 
Table 13. Comparisons of the natural frequencies of pyramidal truss-core sandwich beams with different inclination angles under arbitrary boundary conditions $(\mathrm{Hz})$.

\begin{tabular}{cccccc}
\hline \multirow{2}{*}{$\begin{array}{c}\text { Inclination } \\
\text { Angle }\left(^{(}\right)\end{array}$} & SS-SS & C-C & E3-E3 & E3-SS & E3-C \\
\cline { 2 - 6 } & 124.7301 & 196.8051 & 169.0734 & 148.0808 & 181.0930 \\
15 & 132.9740 & 242.5085 & 193.6238 & 166.4510 & 212.8749 \\
20 & 134.5389 & 266.5863 & 203.0309 & 172.9508 & 226.4270 \\
30 & 133.2646 & 276.5761 & 204.8588 & 173.6883 & 230.2152 \\
40 & 126.4712 & 274.1745 & 197.6094 & 166.8468 & 223.5877 \\
45 & 121.4175 & 266.0154 & 190.4328 & 160.6320 & 215.8123 \\
55 & 107.5508 & 238.4049 & 169.3833 & 142.7266 & 192.2973 \\
65 & 87.0937 & 194.1741 & 137.4324 & 115.7480 & 156.1585 \\
75 & 58.0802 & 129.8441 & 91.7348 & 77.2437 & 104.2799 \\
\hline
\end{tabular}

Table 14. Comparisons of the natural frequencies of tetrahedral truss-core sandwich beams with different inclination angles under arbitrary boundary conditions $(\mathrm{Hz})$.

\begin{tabular}{cccccc}
\hline \multirow{2}{*}{$\begin{array}{c}\text { Inclination } \\
\text { Angle }\left(^{\circ}\right)\end{array}$} & SS-SS & C-C & E3-E3 & E3-SS & E3-C \\
\cline { 2 - 6 } & 116.4407 & 165.7399 & 148.9525 & 132.5366 & 156.6238 \\
20 & 130.5388 & 217.1402 & 181.8840 & 158.1508 & 196.6915 \\
25 & 135.9736 & 251.1696 & 199.1338 & 170.9350 & 219.4220 \\
30 & 137.3951 & 270.8547 & 206.9229 & 176.3563 & 230.5770 \\
40 & 134.6366 & 283.5999 & 208.1194 & 176.2027 & 234.4181 \\
45 & 131.3832 & 281.3719 & 204.3654 & 172.7486 & 230.7946 \\
55 & 120.9219 & 263.9603 & 189.4050 & 159.8195 & 214.5292 \\
65 & 102.8408 & 226.6169 & 161.6207 & 136.2611 & 183.3229 \\
75 & 72.5552 & 160.6354 & 114.2081 & 96.2506 & 129.6388 \\
\hline
\end{tabular}

Table 15. Comparisons of the natural frequencies of 3D-Kagome truss-core sandwich beams with different inclination angles under arbitrary boundary conditions $(\mathrm{Hz})$.

\begin{tabular}{cccccc}
\hline \multirow{2}{*}{$\begin{array}{c}\text { Inclination } \\
\text { Angle }\left(^{(}\right)\end{array}$} & SS-SS & C-C & E3-E3 & E3-SS & E3-C \\
\cline { 2 - 6 } 15 & 127.2862 & 213.6764 & 178.1381 & 154.7157 & 192.9505 \\
20 & 132.3566 & 251.2956 & 196.1763 & 167.8809 & 217.1953 \\
25 & 132.8548 & 268.8284 & 202.2133 & 171.8793 & 226.3097 \\
30 & 131.8481 & 276.0647 & 203.3529 & 172.2663 & 228.8377 \\
40 & 128.7111 & 277.4762 & 200.6993 & 169.5438 & 226.8882 \\
45 & 127.1033 & 275.5196 & 198.5928 & 167.6779 & 224.6977 \\
55 & 124.1497 & 269.3705 & 194.0418 & 163.8221 & 219.5808 \\
65 & 121.4531 & 259.7725 & 188.8268 & 159.6357 & 213.2088 \\
75 & 117.8059 & 237.8299 & 179.1155 & 152.2906 & 200.3804 \\
\hline
\end{tabular}

It can be seen from Tables 10-12 and Figures 12-14 that the variation trend of free vibration frequency curves of structures with different trusses radius is the same under different boundary conditions. In this case, free vibration frequency of the structure first increases and then decreases. The main reasons can be obtained by analyzing Figures 15 and 16. Although both the equivalent density and the equivalent transverse shear modulus increase simultaneously with the increase of the truss radius, when the truss radius is small, the increase in the structural weight is limited but rigidity increases significantly, so it is the control range of rigidity. Hence, the natural frequency increases as the truss radius increases. However, as the truss radius continues to increase, it turns into the control range of structural weight. Then, the free vibration frequencies of the structure will decrease with the increase of the truss radius. 


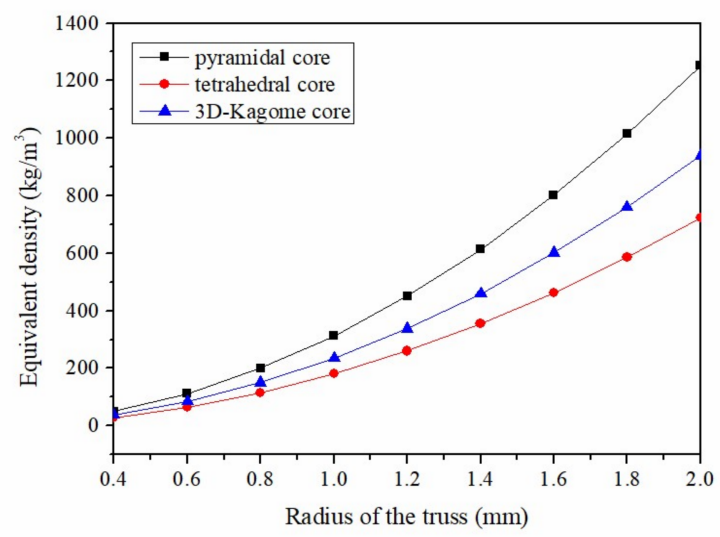

Figure 15. The relationship between truss radius and the equivalent density of the lattice sandwich beam under different truss cores.

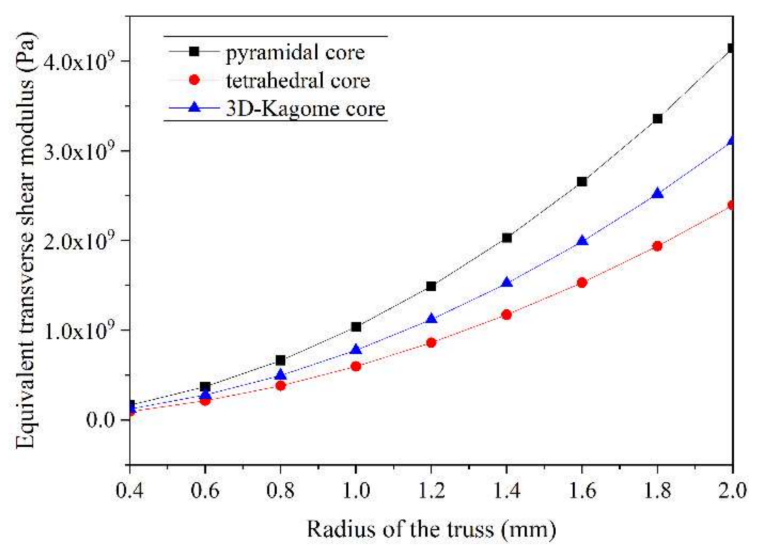

Figure 16. The relationship between truss radius and the equivalent transverse shear modulus of the lattice sandwich beams under different truss cores.

As shown in Tables 13-15 and Figures 17-19, the variation trend of the free vibration frequencies of the structures with different inclination angles is the same under different boundary conditions. In this case, the free vibration frequency of the structure first increases and then decreases. The pyramidal truss core and tetrahedral truss core changes in the same manner. However, for the 3D-Kagome truss core, the mechanism that causes the natural frequency of the structure to change is indeed different. As shown in Figures 20 and 21, as the inclination angle increases, the equivalent density of the 3D-Kagome truss core increases very slowly, but its equivalent transverse shear modulus first increases and then decreases. It is for this reason that, compared with the pyramidal and tetrahedral truss core, the free vibration frequencies of the structure with 3D-Kagome truss core decrease very slowly as the inclination of the truss increases from $15^{\circ}$ to $75^{\circ}$, as shown in Figures 17-19. 


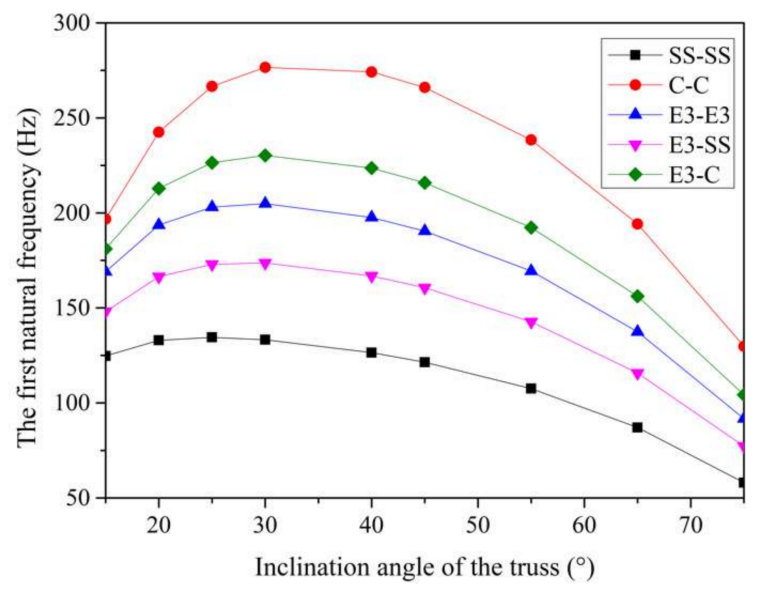

Figure 17. Effect of the inclination angle on the first natural frequency of the pyramidal truss-core sandwich beam under arbitrary boundary conditions.

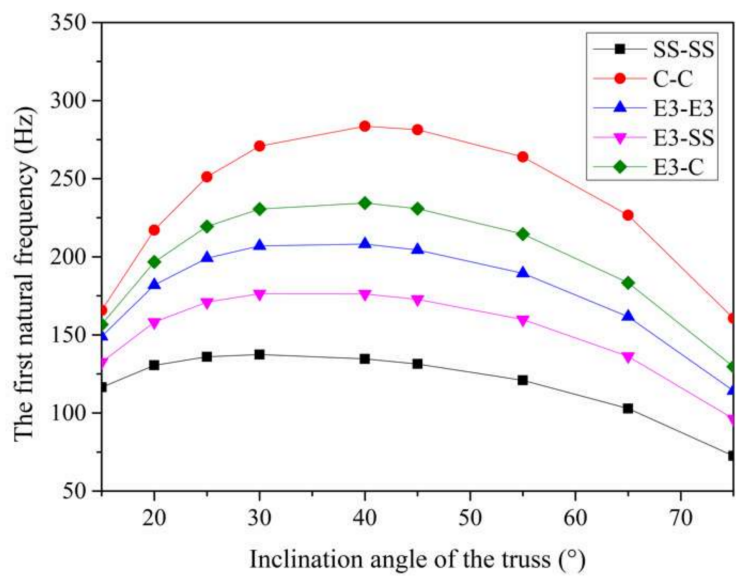

Figure 18. Effect of the inclination angle on the first natural frequency of the tetrahedral truss-core sandwich beam under arbitrary boundary conditions.

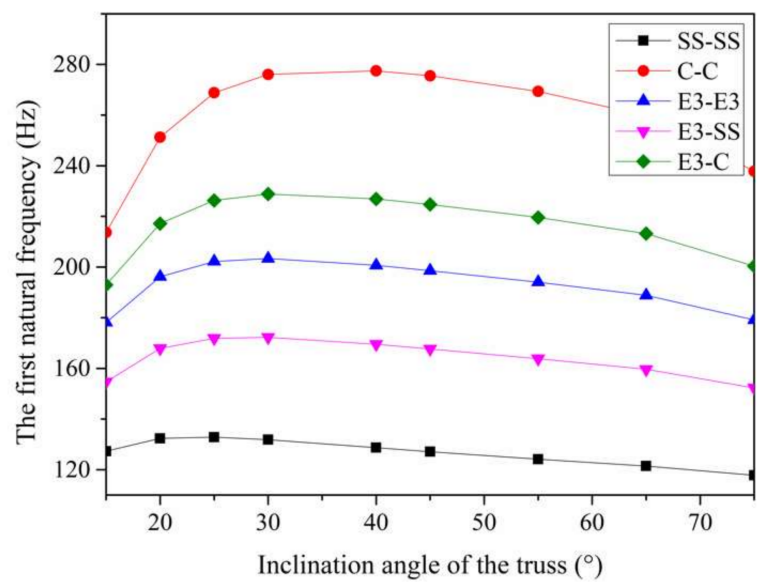

Figure 19. Effect of the inclination angle on the first natural frequency of the 3D-Kagome truss-core sandwich beam under arbitrary boundary conditions. 


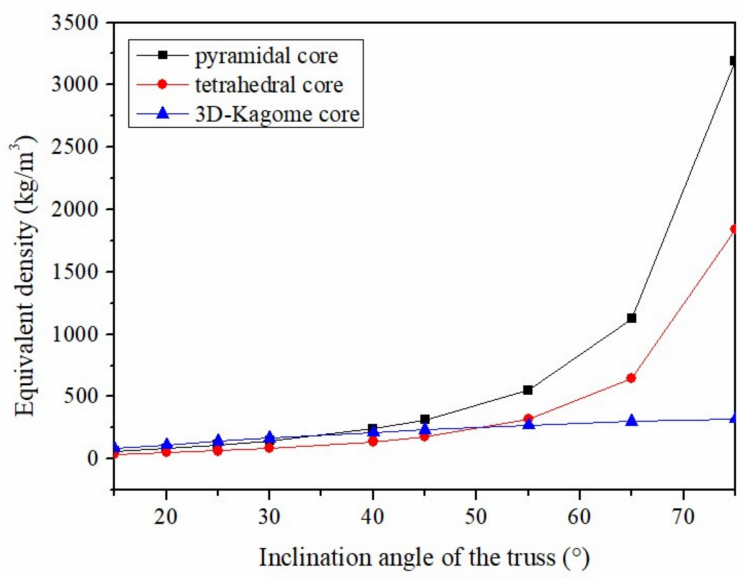

Figure 20. The relationship between the inclination angle and the equivalent density of lattice sandwich beams under different truss cores.

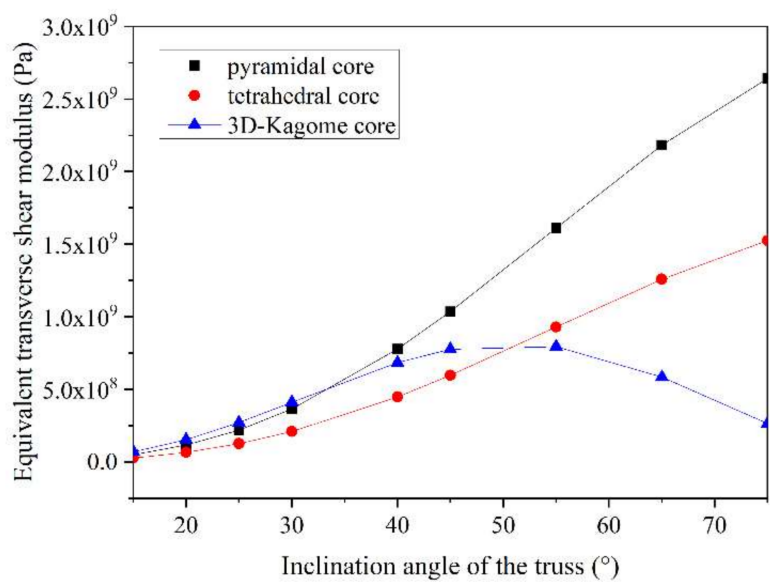

Figure 21. The relationship between the inclination angle and the equivalent transverse shear modulus of lattice sandwich beams under different truss cores.

\subsection{Effect of Damping}

In the above parameter analysis process, damping is not considered, but damping exists in actual structures. Therefore, this section will focus on the vibration characteristics of damped structures. When damping is taken into account, the governing equation of the forced vibration of the lattice sandwich beam can be expressed as follows:

$$
\mathbf{M} \ddot{\mathbf{X}}+\mathbf{C} \dot{\mathbf{X}}+\mathbf{K X}=\mathbf{B}_{0} f(t)
$$

where $\mathbf{M}$ and $\mathbf{K}$ are the mass and rigidity matrices of lattice sandwich beams, respectively, which can be calculated by the expression in Appendix A. C is the viscous damping matrix, $f(t)$ is an external excitation and $\mathrm{B} 0$ is the position vector of the external excitation.

$$
\begin{gathered}
\mathbf{C}=\left(\phi^{T}\right)^{-1} \mathbf{C}_{0}(\phi)^{-1} \\
\mathbf{C}_{0}=\left[\begin{array}{cccc}
2 \omega_{1} \zeta_{1} M_{1} & & \\
& 2 \omega_{2} \zeta_{2} M_{2} \mathbf{M} \phi_{m} & & \\
\vdots & & \ddots & \vdots \\
0 & & \cdots & 2 \omega_{m} \zeta_{m} M_{m}
\end{array}\right]
\end{gathered}
$$


where $C_{0}$ is the generalized damping matrix, $\omega_{m}$ is the $m$ th order natural frequency of lattice sandwich beams, $M_{m}$ is the $m$ th order generalized mass, $\phi$ is the vibration mode matrix, $\phi_{m}$ is the vibration mode vector corresponding to the $m$ th order natural frequency and $\zeta_{m}$ presents the $m$ th order modal damping ratio. Generally, the modal damping ratio needs to be measured according to experimental data. For the convenience of research, $\zeta_{m}$ will be assumed to be 0.005 and 0.01 for all generalized coordinates in this section.

In order to solve the vibration response of lattice sandwich beams subjected to an initial external excitation, it is first necessary to transform Equation (18) into the state space equation form:

$$
\begin{gathered}
\mathbf{Z}=[\dot{\mathbf{X}}, \mathbf{X}] \\
\dot{\mathbf{Z}}=\left[\begin{array}{cc}
0 & \mathbf{I} \\
-\mathbf{M}^{-1} \mathbf{K} & -\mathbf{M}^{-1} \mathbf{C}
\end{array}\right] \mathbf{Z}+\left[\begin{array}{c}
0 \\
-\mathbf{M}^{-1} \mathbf{B}_{0} f(t)
\end{array}\right] \\
\mathbf{B}_{0}=\left[\begin{array}{lll}
\left(\cos \lambda_{r} x_{a}\right)^{T}\left(\sin \lambda_{s} x_{a}\right)^{T} & 0
\end{array}\right]^{T}
\end{gathered}
$$

where $x=\left[w^{T}, \theta^{T}\right]^{T}$ is the generalized coordinate vector, $x_{a}$ represents the external excitation position on the lattice sandwich beam and $r=0, \cdots, M, s=1,2,3,4$.

The natural frequency of the structure remains constant when damping is considered. Therefore, the viscous damping has no effect on the mass and rigidity of the structure. In this section, the transient exciting force of $10 \mathrm{~N}$ lasting for only $0.001 \mathrm{~s}$ is applied on the mid-point of the lattice sandwich beam. Since the force applied is small in magnitude and time, the energy generated is small. Therefore, after the excitation force disappears, the structure will be in a state of free vibration. On this basis, the lsim function in Matlab is used to calculate the time history curve at the mid-point of the lattice sandwich beam. As can be seen in Figures 22 and 23, when damping is not considered, the displacement amplitude of the lattice sandwich beam does not change with time. However, when the influence of damping is considered, the displacement amplitude of the lattice sandwich beam will continue to decrease with time and, the greater the damping, the more the attenuation.

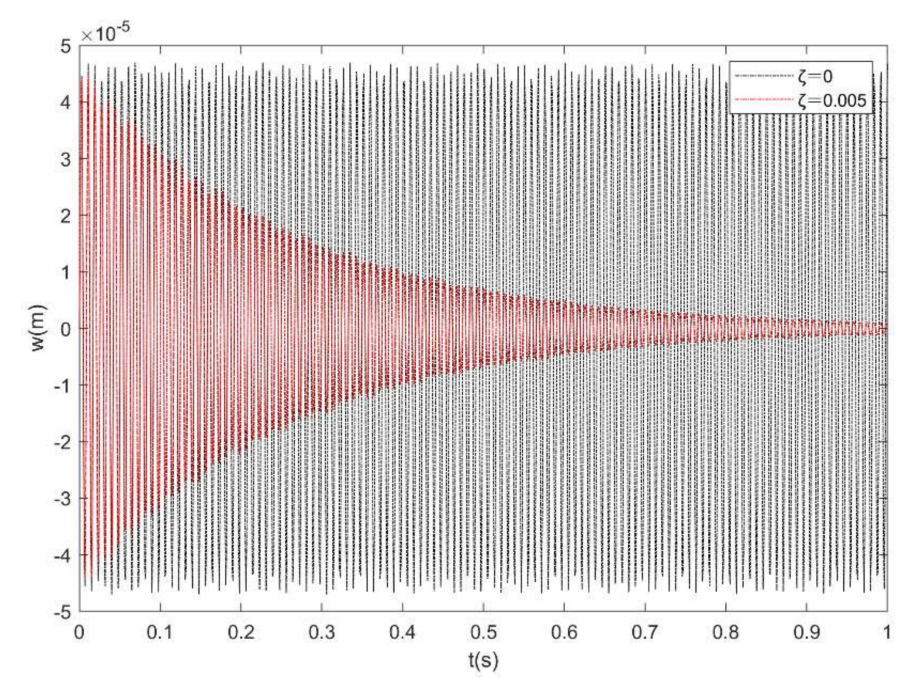

Figure 22. Time-response history at the middle point of the lattice sandwich beam, when $\zeta=0$ and $\zeta=0.005$. 


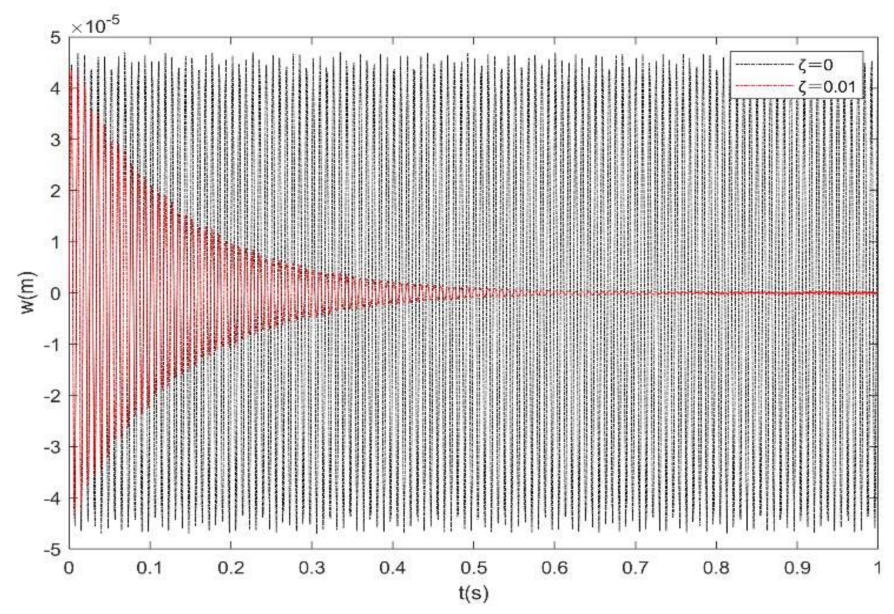

Figure 23. Time-response history at the middle point of the lattice sandwich beam, when $\zeta=0$ and $\zeta=0.01$.

\section{Conclusions}

In this paper, a unified method is proposed to study the free vibration characteristics of lattice sandwich beams under various boundary conditions. In the existing methods, two different types of constraint springs are used to simulate the general elastic support boundary at both ends of lattice sandwich beams. By changing the rigidity of the boundary restraint spring, various boundary conditions can be obtained easily without modifying the solving algorithm and solving process. On this basis, the influence of the structural parameters on the free vibration characteristics of lattice sandwich beams with three different truss cores under different boundary conditions are investigated, including beam length, panel thickness, core height, truss radius, inclination angle and structural damping. From the numerical results, the following conclusions can be drawn:

(1) The analysis result shows that, when the rigidity of the restraint springs is greater than $10^{10}$, it can be regarded as a clamped boundary condition. When the rigidity of the restraint springs is less than $10^{4}$, the support effect of the restraint spring is very weak. It can be regarded as a free boundary condition. When the rigidity of the restraint springs is between $10^{4}$ and $10^{10}$, it can be regarded as an elastic boundary condition.

(2) It can be found that the influence of boundary conditions on structural vibration is very important. As the rigidity of the restraint spring at both ends of the lattice sandwich beam continues to increase, its frequency parameters $(\Omega)$ also increase. Among them, the frequency parameters $(\Omega)$ are the largest under the lamped ends. The elastic boundary support reduces the node effect of the boundary support.

(3) It can be seen that under different boundary conditions, the free vibration frequency of the structure decreases with the increase of beam length but increases with the increase of core height; the free vibration frequencies of the structure will become larger due to the increase of panel thickness under C-C and SS-SS boundary conditions, but it will become smaller as the panel thickness increases under E3-E3, E3-SS and E3-C boundary conditions.

(4) It can be known that with the increase of the truss radius, the free vibration frequency of the structure first increases and then decreases. The main reason is that a smaller radius aids rigidity control, and a larger radius aids quality control. With the increase of the inclination angle, the free vibration frequency of the structure also first increases and then decreases.

(5) It can be learned that when the influence of damping is considered, the displacement amplitude of the lattice sandwich beam will continue to decrease with time and that the greater the damping, the more the attenuation. 
Author Contributions: Conceptualization, Y.J. and R.Y.; methodology, Y.J. and R.Y.; software, H.L. and H.X.; formal analysis, Y.J., R.Y. and H.X.; investigation, H.C. and H.X.; resources, H.L. and H.C.; date curation, Y.J. and R.Y.; writing-original draft preparation, H.L. and H.X.; writing-review and editing, Y.J., R.Y. and H.X.; visualization, H.C. and H.X.; supervision, Y.J. and H.C.; project administration, H.C.; funding acquisition, H.L. All authors have read and agreed to the published version of the manuscript.

Funding: This paper is financially supported by the National Natural Science Foundation of China (Grant No.52071095), the basic research and cutting-edge technology projects of the State Administration for Science Technology and Industry for National Defense (Grant No.JCKY2019604C003) and funds of the State Key Laboratory of Vibration, shock and Noise (6142204190207).

Institutional Review Board Statement: Not applicable.

Informed Consent Statement: Not applicable.

Data Availability Statement: The data used to support the findings of this study are available from the corresponding authors upon request.

Acknowledgments: This paper is financially supported by the National Natural Science Foundation of China (Grant No.52071095), the basic research and cutting-edge technology projects of the State Administration for Science Technology and Industry for National Defense (Grant No.JCKY2019604C003) and funds of the State Key Laboratory of Vibration, shock and Noise (6142204190207).

Conflicts of Interest: The authors declare that they have no conflict of interest.

\section{Appendix A}

According to the above theoretical derivation, both matrix $\mathbf{M}$ and $\mathbf{K}$ are of dimension $2(M+4) \times 2(M+4)$, and vector $A$ has dimension $2(M+4)$. The expression of vector $\mathbf{A}$ is shown as:

$$
\mathbf{A}=\left\{A_{0}, A_{1}, \ldots, A_{m}, \ldots A_{M}, A_{1}^{a}, A_{2}^{a}, A_{3}^{a}, A_{4}^{a}, B_{0}, B_{1}, \ldots, B_{m}, \ldots B_{M}, B_{1}^{a}, B_{2}^{a}, B_{3}^{a}, B_{4}^{a}\right\}
$$

In Equation (17), the expressions of the mass matrix $\mathbf{M}$ and the stiffness matrix $\mathbf{K}$ for the lattice sandwich beam with arbitrary boundary conditions can be given separately as:

$$
\begin{gathered}
\mathbf{K}=\mathbf{K}_{P}+\mathbf{K}_{S} \\
\mathbf{K}_{P}=\left[\begin{array}{cccc}
K_{11}^{P} & K_{12}^{P} & K_{13}^{P} & K_{14}^{P} \\
K_{21}^{P} & K_{22}^{P} & K_{23}^{P} & K_{24}^{P} \\
K_{31}^{P} & K_{32}^{P} & K_{33}^{P} & K_{34}^{P} \\
K_{41}^{P} & K_{42}^{P} & K_{43}^{P} & K_{44}^{P}
\end{array}\right] \\
K_{11}^{P}=B\left(D_{\mathrm{t}}+D_{b}\right) \lambda_{r}^{2} \lambda_{i}^{2} \int_{0}^{L} \cos \lambda_{r} x \cos \lambda_{i} x d x \\
K_{33}^{P}=B\left(A_{\mathrm{t}}+A_{b}\right) \lambda_{r} \lambda_{i} \int_{0}^{L} \sin \lambda_{r} x \sin \lambda_{i} x d x+B A_{\mathrm{c}} \int_{0}^{L} \cos \lambda_{r} x \cos \lambda_{i} x d x \\
K_{44}^{P}=B\left(D_{\mathrm{t}}+A_{b}\right) \lambda_{\mathrm{s}} \lambda_{j} \lambda_{0}^{2} \int_{0}^{L} \sin \lambda_{s} x \sin \lambda_{j} x d x \\
K_{12}^{P}=B\left(D_{\mathrm{t}}+D_{b}\right) \lambda_{r}^{2} \lambda_{\mathrm{j}}^{2} \int_{0}^{L} \cos \lambda_{j} x d x+B A_{c} \int_{0}^{L} \sin \lambda_{s} x \sin \lambda_{j} x d x \\
K_{13}^{P}=B\left(-B_{\mathrm{t}}+B_{b}\right) \lambda_{r}^{2}\left(-\lambda_{i}\right) \int_{0}^{L} \cos \lambda_{r} x \sin \lambda_{i} x d x \\
K_{14}^{P}=B\left(-B_{\mathrm{t}}+B_{b}\right) \lambda_{r}^{2} \lambda_{j} \int_{0}^{L} \cos \lambda_{r} x \cos \lambda_{j} x d x
\end{gathered}
$$




$$
\begin{aligned}
& K_{23}^{P}=B\left(-B_{\mathrm{t}}+B_{b}\right) \lambda_{s}^{2}\left(-\lambda_{i}\right) \int_{0}^{L} \sin \lambda_{s} x \sin \lambda_{i} x d x \\
& K_{24}^{P}=B\left(-B_{\mathrm{t}}+B_{b}\right) \lambda_{s}^{2} \lambda_{j} \int_{0}^{L} \sin \lambda_{s} x \cos \lambda_{j} x d x \\
& K_{34}^{P}=B\left(A_{\mathrm{t}}+A_{b}\right)\left(-\lambda_{r}\right) \lambda_{j} \int_{0}^{L} \sin \lambda_{r} x \cos \lambda_{j} x d x+B A_{\mathrm{c}} \int_{0}^{L} \cos \lambda_{r} x \sin \lambda_{j} x d x \\
& K_{21}^{P}=\left(K_{12}^{P}\right)^{T}, K_{31}^{P}=\left(K_{13}^{P}\right)^{T}, K_{41}^{P}=\left(K_{14}^{P}\right)^{T}, K_{32}^{P}=\left(K_{23}^{P}\right)^{T}, K_{42}^{P}=\left(K_{24}^{P}\right)^{T}, K_{43}^{P}=\left(K_{34}^{P}\right)^{T} \\
& \mathbf{K}_{S}=\left[\begin{array}{cccc}
K_{11}^{S} & 0 & 0 & 0 \\
0 & K_{22}^{S} & 0 & 0 \\
0 & 0 & K_{33}^{S} & 0 \\
0 & 0 & 0 & 0
\end{array}\right] \\
& K_{11}^{S}=B\left(k_{0}+(-1)^{r+i} k_{L}\right) \\
& K_{22}^{S}=B\left(K_{0} \lambda_{s} \lambda_{j}+(-1)^{s+j} \lambda_{s} \lambda_{j} K_{L}\right) \\
& K_{33}^{S}=B\left(K_{0}+(-1)^{r+i} K_{L}\right) \\
& \mathbf{M}=\left[\begin{array}{llll}
M_{11} & M_{12} & M_{13} & M_{14} \\
M_{21} & M_{22} & M_{23} & M_{24} \\
M_{31} & M_{32} & M_{33} & M_{34} \\
M_{41} & M_{42} & M_{43} & M_{44}
\end{array}\right] \\
& M_{11}=B\left(I_{3}^{t}+I_{3}^{b}+I_{1}^{c}\right) \lambda_{r} \lambda_{i} \int_{0}^{L} \sin \lambda_{r} x \sin \lambda_{i} x d x+B\left(I_{4}^{t}+I_{4}^{b}+I_{2}^{c}\right) \int_{0}^{L} \cos \lambda_{r} x \cos \lambda_{i} x d x \\
& M_{22}=B\left(I_{3}^{t}+I_{3}^{b}+I_{1}^{c}\right) \lambda_{s} \lambda_{j} \int_{0}^{L} \cos \lambda_{s} x \cos \lambda_{j} x d x+B\left(I_{4}^{t}+I_{4}^{b}+I_{2}^{c}\right) \int_{0}^{L} \sin \lambda_{s} x \sin \lambda_{j} x d x \\
& M_{33}=B\left(I_{1}^{t}+I_{1}^{b}+I_{1}^{c}\right) \lambda_{s} \lambda_{j} \int_{0}^{L} \cos \lambda_{r} x \cos \lambda_{i} x d x \\
& M_{44}=B\left(I_{1}^{t}+I_{1}^{b}+I_{1}^{c}\right) \lambda_{s} \lambda_{j} \int_{0}^{L} \sin \lambda_{s} x \sin \lambda_{j} x d x \\
& M_{12}=B\left(I_{3}^{t}+I_{3}^{b}+I_{1}^{c}\right)\left(-\lambda_{r}\right) \lambda_{j} \int_{0}^{L} \sin \lambda_{r} x \cos \lambda_{j} x d x+B\left(I_{4}^{t}+I_{4}^{b}+I_{2}^{c}\right) \int_{0}^{L} \cos \lambda_{r} x \sin \lambda_{j} x d x \\
& M_{13}=B\left(I_{2}^{t}-I_{2}^{b}-I_{1}^{c}\right)\left(-\lambda_{r}\right) \int_{0}^{L} \sin \lambda_{r} x \cos \lambda_{i} x d x \\
& M_{14}=B\left(I_{2}^{t}-I_{2}^{b}-I_{1}^{c}\right)\left(-\lambda_{r}\right) \int_{0}^{L} \sin \lambda_{r} x \sin \lambda_{j} x d x \\
& M_{23}=B\left(I_{2}^{t}-I_{2}^{b}-I_{1}^{c}\right) \lambda_{s} \int_{0}^{L} \cos \lambda_{s} x \cos \lambda_{i} x d x \\
& M_{24}=B\left(I_{2}^{t}-I_{2}^{b}-I_{1}^{c}\right)\left(-\lambda_{r}\right) \int_{0}^{L} \cos \lambda_{s} x \sin \lambda_{j} x d x \\
& M_{34}=B\left(I_{1}^{t}+I_{1}^{b}+I_{1}^{c}\right)\left(-\lambda_{r}\right) \int_{0}^{L} \cos \lambda_{r} x \sin \lambda_{j} x d x \\
& M_{21}=\left(M_{12}\right)^{T}, M_{31}=\left(M_{13}\right)^{T}, M_{41}=\left(M_{14}\right)^{T}, M_{32}=\left(M_{23}\right)^{T}, M_{42}=\left(M_{24}\right)^{T}, M_{43}=\left(M_{34}\right)^{T} \\
& \text { where } r, i=0, \cdots,+\infty, s, j=1,2,3,4 \text {. }
\end{aligned}
$$

\section{References}

1. Ashby, M. Drivers for material development in the 21st century. Prog. Mater. Sci. 2001, 46, 191-199. [CrossRef]

2. Evans, A.; Hutchinson, J.; Fleck, N.; Ashby, M.; Wadley, H. The topological design of multifunctional cellular metals. Prog. Mater. Sci. 2001, 46, 309-327. [CrossRef] 
3. Wallach, J.; Gibson, L. Mechanical behavior of a three-dimensional truss material. Int. J. Solids Struct. 2001, 38, 7181-7196. [CrossRef]

4. Vasiliev, V.; Razin, A. Anisogrid composite lattice structures for spacecraft and aircraft applications. Compos. Struct. 2006, 76, 182-189. [CrossRef]

5. Lu, T.; Valdevit, L.; Evans, A. Active cooling by metallic sandwich structures with periodic cores. Prog. Mater. Sci. 2005, 50, 789-815. [CrossRef]

6. Vaidya, A.; Vaidya, U.; Uddin, N. Impact response of three-dimensional multifunctional sandwich composite. Mater. Sci. Eng. A 2008, 472, 52-58. [CrossRef]

7. Fan, H.; Jin, F.; Fang, D. Mechanical properties of hierarchical cellular materials. Part I: Analysis. Compos. Sci. Technol. 2008, 68, 3380-3387. [CrossRef]

8. Queheillalt, D.T.; Wadley, H.N. Titanium alloy lattice truss structures. Mater. Des. 2009, 30, 1966-1975. [CrossRef]

9. Wadley, H.N.; Fleck, N.A.; Evans, A.G. Fabrication and structural performance of periodic cellular metal sandwich structures. Compos. Sci. Technol. 2003, 63, 2331-2343. [CrossRef]

10. Deshpande, V.; Ashby, M.; Fleck, N. Foam topology: Bending versus stretching dominated architectures. Acta Mater. 2001, 49, 1035-1040. [CrossRef]

11. Rizov, V.; Shipsha, A.; Zenkert, D. Indentation study of foam core sandwich composite panels. Compos. Struct. 2005, 69, 95-102. [CrossRef]

12. Rathbun, H.; He, M.; Ferri, E.; Mercer, C.; Mcmeeking, R.M.; Evans, A.G. Structural performance of metallic sandwich panels with square honeycomb cores. Philos. Mag. 2005, 85, 3207-3234. [CrossRef]

13. Pan, S.-D.; Wu, L.-Z.; Sun, Y.-G.; Zhou, Z.-G. Fracture test for double cantilever beam of honeycomb sandwich panels. Mater. Lett. 2008, 62, 523-526. [CrossRef]

14. Xiong, J.; Ma, L.; Wu, L.Z.; Wang, B.; Vaziri, A. Fabrication and crushing behavior of low density carbon fiber composite py-ramidal truss structures. Compos. Struct. 2010, 92, 2695-2702. [CrossRef]

15. Queheillalt, D.T.; Wadley, H.N. Pyramidal lattice truss structures with hollow trusses. Mater. Sci. Eng. A 2005, 397, 132-137. [CrossRef]

16. Zok, F.; Waltner, S.; Wei, Z.; Rathbun, H.; McMeeking, R.; Evans, A. A protocol for characterizing the structural performance of metallic sandwich panels: Application to pyramidal truss cores. Int. J. Solids Struct. 2004, 41, 6249-6271. [CrossRef]

17. Biagi, R.; Bart-Smith, H. Imperfection sensitivity of pyramidal core sandwich structures. Int. J. Solids Struct. 2007, 44, 4690-4706. [CrossRef]

18. Cote, F.; Biagi, R.; Bart-Smith, H.; Deshpande, V.S. Structural response of pyramidal core sandwich columns. Int. J. Solids Struct. 2007, 44, 3533-3556. [CrossRef]

19. Kooistra, G.W.; Deshpande, V.S.; Wadley, H.N. Compressive behavior of age hardenable tetrahedral lattice truss struc-tures made from aluminum. Acta Mater. 2004, 52, 4229-4237. [CrossRef]

20. Zhang, G.; Ma, L.; Wang, B.; Wu, L. Mechanical behaviour of CFRP sandwich structures with tetrahedral lattice truss cores. Compos. Part B Eng. 2012, 43, 471-476. [CrossRef]

21. Hwang, J.S.; Choi, T.G.; Lee, D.; Lyu, M.Y.; Yang, D.Y. Dynamic and static characteristics of polypropylene py-ramidal kagome structures. Compos. Struct. 2015, 131, 17-24. [CrossRef]

22. Lee, Y.-H.; Lee, B.-K.; Jeon, I.; Kang, K.-J. Wire-woven bulk Kagome truss cores. Acta Mater. 2007, 55, 6084-6094. [CrossRef]

23. Yang, F.; Cheng, S.; Zeng, T.; Wang, Z.-H.; Xu, G.-D.; Zhai, J.-J.; Fang, D.-N. Mechanical and oxidation properties of C/SiC corrugated lattice core composite sandwich panels. Compos. Struct. 2016, 158, 137-143. [CrossRef]

24. Song, Z.-Z.; Cheng, S.; Zeng, T.; Yang, F.; Jing, S.-D.; Fang, D.-N. Compressive behavior of C/SiC composite sandwich structure with stitched lattice core. Compos. Part B Eng. 2015, 69, 243-248. [CrossRef]

25. Deshpande, V.; Fleck, N. Collapse of truss core sandwich beams in 3-point bending. Int. J. Solids Struct. 2001, 38, 6275-6305. [CrossRef]

26. Hwu, C.; Chang, W.; Gai, H. Vibration suppression of composite sandwich beams. J. Sound Vib. 2004, 272, 1-20. [CrossRef]

27. Lou, J.; Ma, L.; Wu, L.-Z. Free vibration analysis of simply supported sandwich beams with lattice truss core. Mater. Sci. Eng. B 2012, 177, 1712-1716. [CrossRef]

28. Lou, J.; Wang, B.; Ma, L.; Wu, L.Z. Free vibration analysis of lattice sandwich beams under several typical boundary condi-tions. Acta Mech. Solida Sin. 2013, 26, 458-467. [CrossRef]

29. Xu, M.; Qiu, Z. Free vibration analysis and optimization of composite lattice truss core sandwich beams with interval parameters. Compos. Struct. 2013, 106, 85-95. [CrossRef]

30. Chen, J.E.; Zhang, W.; Sun, M.; Yao, M.H. Free Vibration and Hardening Behavior of Truss Core Sandwich Beam. Shock. Vib. 2016, 2016, 7348518. [CrossRef]

31. Li, F.-M.; Lyu, X.-X. Active vibration control of lattice sandwich beams using the piezoelectric actuator/sensor pairs. Compos. Part B Eng. 2014, 67, 571-578. [CrossRef]

32. Zhao, Z.; Wen, S.; Li, F. Vibration analysis of multi-span lattice sandwich beams using the assumed mode method. Compos. Struct. 2018, 185, 716-727. [CrossRef]

33. Xu, G.D.; Zeng, T.; Cheng, S.; Wang, X.H.; Zhang, K. Free vibration of composite sandwich beam with graded corrugated lat-tice core. Compos. Struct. 2019, 229, 334-344. [CrossRef] 
34. Li, W. Free Vibrations of Beams with General Boundary Conditions. J. Sound Vib. 2000, 237, 709-725. [CrossRef]

35. Li, W. Vibration analysis of rectangular plates with general elastic boundary supports. J. Sound Vib. 2004, 273, 619-635. [CrossRef]

36. Zhang, X.; Li, W.L. Vibrations of rectangular plates with arbitrary non-uniform elastic edge restraints. J. Sound Vib. 2009, 326, 221-234. [CrossRef]

37. Khov, H.; Li, W.L.; Gibson, R.F. An accurate solution method for the static and dynamic deflections of orthotropic plates with general boundary conditions. Compos. Struct. 2009, 90, 474-481. [CrossRef]

38. Shi, D.; Wang, Q.; Shi, X.; Pang, F. An accurate solution method for the vibration analysis of Timoshenko beams with general elastic supports. Proc. Inst. Mech. Eng. Part C J. Mech. Eng. Sci. 2014, 229, 2327-2340. [CrossRef]

39. Wang, Q.; Shi, D.; Liang, Q. Free vibration analysis of axially loaded laminated composite beams with general boundary conditions by using a modified Fourier-Ritz approach. J. Compos. Mater. 2016, 50, 2111-2135. [CrossRef]

40. Pang, F.; Li, H.; Du, Y.; Li, S.; Chen, H.; Liu, N. A Series Solution for the Vibration of Mindlin Rectangular Plates with Elastic Point Supports around the Edges. Shock. Vib. 2018, 2018, 8562079. [CrossRef]

41. Li, H.; Liu, N.; Pang, F.; Du, Y.; Li, S. An Accurate Solution Method for the Static and Vibration Analysis of Functionally Graded Reissner-Mindlin Rectangular Plate with General Boundary Conditions. Shock. Vib. 2018, 2018, 4535871. [CrossRef]

42. Li, S.; Fan, H. Flexural behaviors and local failure analyses of EPS foam-filled GFRC truss-core sandwich panels. Case Stud. Constr. Mater. 2021, 16, e00688. [CrossRef]

43. Guo, Z.-K.; Hu, G.; Sorokin, V.; Yang, Y.; Tang, L. Sound transmission through sandwich plate with hourglass lattice truss core. J. Sandw. Struct. Mater. 2021, 23, 1902-1928. [CrossRef]

44. Yang, J.-S.; Chen, S.-Y.; Li, S.; Pang, Y.-Z.; Schmidt, R.; Schröder, K.-U.; Qu, J.; Wu, L.-Z. Dynamic responses of hybrid lightweight composite sandwich panels with aluminium pyramidal truss cores. J. Sandw. Struct. Mater. 2021, 23, 2176-2195. [CrossRef]

45. Wu, H.; Zhu, H.; Chen, L.; Pan, D.; Fang, Q.; Li, W.; Jin, F.; Fan, H. Engineering failure analyses of aluminum truss-core sandwich panels of portable deployable roadway under tracked loads. Eng. Fail. Anal. 2021, 128, 105655. [CrossRef]

46. Sun, L.; Li, J.; Xiao, Y. Broad and Low Frequency Bandgap in Truss Core Sandwich Beam. Mech. Solids 2021, 56, 421-429. [CrossRef] 